\title{
Computerlinguistische Verfahren zur Vermessung wissenschaftsdisziplinärer Kommunikation. Eine exemplarische Studie über die Berufs- und Wirtschaftspädagogik
}

\author{
Robert W. Jahn $(D)$ Jan Goldenstein • Mathias Götzl
}

Eingegangen: 22. September 2020 / Überarbeitet: 1. März 2021 / Angenommen: 15. April 2021 / Online publiziert: 24. August 2021

(C) Der/die Autor(en) 2021

Zusammenfassung Wissenschaftliche Disziplinen als Kommunikations- und Diskursgemeinschaften spezieller Fachleute befassen sich nicht allein mit ihren Gegenständen sowie der eigenen Methodologie und Methodik, sondern auch mit sich selbst und der Entwicklung ihrer paradigmatischen Grundlagen. Dies hat auch in den Erziehungswissenschaften eine breite Tradition. Zur Analyse paradigmatischer Entwicklungen der Ideen- und Sozialgestalt einer Disziplin eignet sich insbesondere die Untersuchung der disziplinären Kommunikation. Interessant ist in diesem Zusammenhang vor allem die formelle (schriftliche) Kommunikation, die in disziplinären Fachzeitschriften erfolgt.

Qualitative Analysen sind jedoch sehr aufwändig, insb. wenn größere Datenmengen und Zeiträume analysiert werden sollen. Zudem besteht das Problem, dass eine deduktive, thematische Analyse disziplinäre Kartierungsansätze braucht, die relativ trennscharf die disziplinären Gegenstände operationalisieren. Demgegenüber erlauben quantitativ orientierte computerlinguistische Analysen einen induktiven Zugriff auf die in Zeitschriften publizierten Themen sowie die Verarbeitung großer Datenmengen. In diesem Beitrag wird ein computerlinguistisches topic-modeling-Verfah-

Prof. Dr. Robert W. Jahn ( $₫)$

Fakultät für Humanwissenschaften, Otto-von-Guericke-Universität Magdeburg,

Zschokkestr. 32, 39104 Magdeburg, Deutschland

E-Mail: robert.jahn@ovgu.de

Dr. Jan Goldenstein

Lehrstuhl für Organisation, Führung und Human Ressource Management,

Wirtschaftswissenschaftliche Fakultät, Friedrich-Schiller-Universität Jena,

Carl-Zeiß-Straße 3, 07743 Jena, Deutschland

E-Mail: jan.goldenstein@uni-jena.de

Dr. Mathias Götzl

Staatliches Studienseminar für Lehrerausbildung Erfurt - Lehramt an berufsbildenden Schulen,

Erfurt, Deutschland

E-Mail: mathias.goetzl@ netzwerk-bwp.de 
ren vorgestellt und angewandt, dass die Themen einer gesamten Zeitschrift induktiv extrahiert und jeden ihrer einzelnen Beiträge quantitativ thematisch gewichtet.

Dies erfolgt am Beispiel der Berufs- und Wirtschaftspädagogik als eine erziehungswissenschaftliche Teildisziplin und der „Berufs- und Wirtschaftspädagogik online“" als einer ihrer zentralen Fachzeitschriften. Diese hat zwei wesentliche Vorteile als Anwendungsbeispiel. Erstens liegt sie bereits in digitalisierter Form vor. Zweitens eignet sie sich zur Validierung des Verfahrens und der identifizierten Themen, da ihre einzelnen Ausgaben bereits themenbezogen sind.

Es kann gezeigt werden, dass die untersuchte Zeitschrift zentrale Forschungsgegenstände der Disziplin abbildet. Das Verfahren eignet sich zudem auch, um methodische und methodologische Topics in den Beiträgen zu identifizieren. Es lässt darüber hinaus eine Verbindung zwischen der kognitiv-inhaltlichen und sozialen Dimension paradigmatischer Entwicklungen zu, da Ähnlichkeiten und Unterschiede in der thematischen Kommunikation zwischen Personen und Organisationen ermittelt werden können. So können z. B. sechs Gruppen von Universitätsstandorten identifiziert werden, die inhaltlich an ähnlichen Themen arbeiten.

Schlüsselwörter Wissenschaftsforschung · Fachzeitschriften · Berufs- und Wirtschaftspädagogik · Computerlinguistik · Topic-Modeling

\title{
Computational linguistic methods for measuring scientific communication. Study of vocational and business education using the example of one of their journals
}

\begin{abstract}
Scientific disciplines as communication and discourse communities of special experts deal not only with their subjects and their own methodology and methods, but also with themselves and the development of their paradigmatic foundations. This also has a broad tradition in educational science. The examination of discipline communication is particularly suitable for analyzing paradigmatic developments of cognitive and social dimension of science. In this context, the formal (written) communication that takes place in disciplinary journals is particularly interesting.
\end{abstract}

However, qualitative analyzes are very time-consuming, especially when larger amounts of data and time periods are to be analyzed. In addition, there is the problem that a deductive, topic analysis needs disciplinary mapping approaches that operationalize the discipline objects relatively clearly. In contrast, quantitatively oriented computational linguistic analyzes allow inductive access to the topics published in journals and the processing of large amounts of data. In this article, a computer linguistic topic-modeling process is presented and applied, which inductively extracts the topics of an entire journal and gives each of its individual articles a quantitative topic weighting.

This is done using the example of vocational and business education as an educational sub-discipline and "Berufs- und Wirtschaftspädagogik—online" as one of its central specialist journals. This has two main advantages as an application example. First, it is already available in digitized form. Second, it is suitable for validating the process and the identified topics, as its several issues are already topic-related. 
Our study shows that the investigated journal captures central research subjects of the scientific discipline. The procedure applied is also suitable for identifying methodical and methodological topics in the articles. Furthermore, as similarities and differences in thematic communication between people and organizations can be determined, the procedure also allows us to link the cognitive-content and social dimensions of paradigmatic progress. For example, we identified six groups of universities that work on similar topics.

Keywords Science research · Scientific journals · Vocational and business education $\cdot$ Computational linguistics $\cdot$ Topic modeling

\section{Einleitung}

Wissenschaftliche Disziplinen sind keine monolithischen, starren Systeme, sondern dynamische, fluide Strukturen, die durch Prozesse von Wandel, Angleichung und Differenzierung geprägt sind (Kuhn 2014, S. 63). Sie durchlaufen einen Entwicklungsprozess von den bescheidenen Anfängen als (akademische) Idee hin zu einer institutionalisierten Erkenntnisinstanz (Clark 1972). Aus der Perspektive der soziologischen Feldtheorie lassen sie sich beschreiben als institutionelle Felder, in denen ein gemeinsames Interesse kodifiziert wird und die zugleich Strukturen im Sinne von Interaktions- und Kommunikationsformen, Teilnahmebedingungen oder Regeln prägen. Feldinteressen und -strukturen sind ebendann auch Gegenstand von Aushandlungsprozessen und Machtkämpfen und bedingen Wandlungs- und Entwicklungsmöglichkeiten der Disziplin und ihrer Akteure (Bourdieu 1984; Martin 2003; Zietsma et al. 2017). In ähnlicher Form begreift Kuhn (1962, 2014) disziplinäre Entwicklungsprozesse nicht als kumulative Anhäufung von Erkenntnissen, sondern als wechselnde Abfolge aus paradigmatischen Krisen und Phasen normaler Wissenschaft. Paradigmen (der Normal Science) beschreiben nach Kuhn ein allgemein anerkanntes Forschungsprogramm (Feldinteresse) mit etablierten Theorien und Methoden, bewährten Forschungsansätzen und Problemlösungen (Feldstrukturen). Neben dieser epistemologischen Ebene des Begriffs hat Paradigma aber auch eine soziale Bedeutungsdimension, die mit der ersten interdependent verbunden ist. Paradigmen stehen gleichermaßen für eine Gruppe von Wissenschaftler*innen, die mit ähnlichen Methoden an ähnlichen Fragestellungen arbeiten (Felt et al. 1995, S. 291). Entwicklungen wissenschaftlicher Disziplinen vollziehen sich demnach auf mindestens zwei interdependenten Ebenen: der Sozial- und Ideengestalt (Averbeck 2008; Kaesler 1984), welche auch als soziale und kognitive Orientierungen (Felt et al. 1995, S. 126) bzw. als die soziale und kognitive Identität (Lepenies 1981) einer Disziplin bezeichnet werden.

Versteht man Disziplinen als institutionelle Felder, so spiegelt sich ihre Ideengestalt im Inhalt (Botschaften) der Kommunikation, ihre Sozialgestalt in den Sendern und Empfängern der Kommunikation wider. Kommunikationsprozesse einer Disziplin können damit Aufschluss geben über ihre Verfassung und Entwicklung - und das sowohl aus der Perspektive einer einzeldisziplinären Wissenschaftsforschung, die auf Selbstvergewisserung abzielt, als auch aus der Perspektive einer allgemei- 
nen Wissenschaftsforschung, die auf (verstehende) Deskription und Explikation der Entwicklung wissenschaftlicher Disziplinen, Felder oder Systeme fokussiert.

Für die erziehungswissenschaftliche Teildisziplin „Berufs- und Wirtschaftspädagogik", die in diesem Beitrag als Anwendungsbeispiel dienen wird, stellt Reinisch (2009) fest, dass sie als ,etablierte Wissenschaft“ im Sinne von Clark (1972, 1974) bezeichnet werden kann und als „,normale Wissenschaft“ im Sinne Kuhns (1962, 2014) betrieben wird. Während die Etablierungsthese auch empirisch gestützt werden kann (Götzl et al. 2018), ist seine Einschätzung, die BWP werde als normale Wissenschaft betrieben, vor dem Hintergrund disziplinärer Pluralität in den methodologischen Ansätzen (s.a. Zabeck 1978) kritisch zu reflektieren und empirisch zu prüfen. Reinisch und Zabeck resümieren zugleich, dass Disziplinen, die einen solchen Status erlangt haben, immer wieder selbst ihre paradigmatischen Grundlagen reflektieren müssen (Reinisch 2009). An diesem innerdisziplinären Desiderat setzt dieser Beitrag folglich einerseits an. In einem weiteren Kontext geht es andererseits darum, methodische und technische Möglichkeiten empirisch fundierter Analysen der sozialen und kognitiven Verfassung bzw. der Entwicklung der Sozial- und Ideengestalt wissenschaftlicher Disziplinen vorzustellen und (am Beispiel der Berufsund Wirtschaftspädagogik) zu erproben.

Vor diesem Hintergrund soll die Kommunikation in Fachzeitschriften (als bedeutende Medien wissenschaftlicher Kommunikation) in den Mittelpunkt der Betrachtung gestellt werden. Dabei liegt der Fokus einerseits auf einer sozialen und inhaltlichen Ebene und hierbei auf der Frage, wer worüber kommuniziert (soziale und kognitive Dimension). Andererseits liegt der Fokus auf der methodischen Ebene und der Frage, ob sich computerlinguistische Verfahren zur Analyse disziplinärer Kommunikation eignen.

Für die Berufs- und Wirtschaftspädagogik liegen bereits einige wenige, erste (Partial-)Analysen zur disziplinären Kommunikation in Zeitschriften vor. Diese beziehen sich erstens auf die Frage, welche Zeitschriften für die disziplinäre Kommunikation relevant sind und welche (reputierliche) Bedeutung diese haben (z. B. Baumann 2009; Klusmeyer 2001, S. 34 ff.; Klusmeyer et al. 2011; Reinisch 2006; Söll et al. 2014). Zweitens befassen sich einige der Analysen explizit mit der ,Zeitschrift für Berufs- und Wirtschaftspädagogik“ (ZBW) als „Leitmedium“ resp. „Flaggschiff“ der Disziplin (Klusmeyer 2001, S. 39; Pätzold 2004, S. 484; Reinisch 2006, S. 161; Tenberg et al. 2009, S. 419; Zabeck 1998, S. 174) und analysieren (ausgewählte) Aspekte ihrer Entwicklung auf formaler, sozialer und/oder inhaltlicher Ebene bzw. ihre Verwissenschaftlichung (z.B. Harney et al. 1994; Jahn et al. 2019; Kipp und Miller-Kipp 2004; Klusmeyer 1999, 2001, 2002a, b, 2010; Pätzold 2004; Stratmann 1992; Tenberg et al. 2009). Probleme bereitet dabei insb. die thematische Analyse und mithin die Frage nach dem Inhalt der disziplinären Kommunikation (als Gegenstand der Disziplin) sowie Möglichkeiten einer induktiven oder deduktiven, möglichst objektiven, quantifizierbaren und multithematischen Codierung einzelner Artikel. Diese Defizite liefern Argumente für einen Zugriff, der mit computerlinguistischen Verfahren möglich wird. Diese Verfahren (wie sie z. B. Zawacki-Richter und Naidu (2016) oder Goldenstein und Poschmann (2019a, b) nutzen) ermöglichen nicht nur einen induktiven Zugriff auf große Datenmengen, sie sind zudem objektiv, reliabel und erfolgt systematisch und standardisiert. 
Im Beitrag wird die Fragestellung untersucht, ob das im Weiteren vorgestellte Verfahren geeignet ist, thematische Orientierungen und Konjunkturen einer Disziplin (als Ausdruck der Ideengestalt) objektiv abzubilden und diese in Beziehung zur Sozialgestalt zu setzen. Durch diese Verbindung von Ideen- und Sozialgestalt ließen sich die dynamischen Strukturen erfassen und Prozesse von Wandel, Angleichung und Differenzierung innerhalb institutioneller Felder nachzeichnen. Um dieses Verfahren zu testen, ist die hier exemplarisch ausgewählte Fachzeitschrift „Berufs- und Wirtschaftspädagogik - online“ (bwp@) in besonderer Weise geeignet - nicht so sehr, weil sie als Online-Zeitschrift bereits digital vorliegt, sondern weil ihre Ausgaben thematisch fokussiert sind. Die computerlinguistischen Analysen müssen, wenn sie valide Ergebnisse erzeugen sollen, verschiedene thematische Fokusse adäquat abbilden.

Dazu wird im Beitrag zunächst in die (disziplinäre) Wissenschaftsforschung eingeführt sowie auf die Rolle von Fachzeitschriften und die Stellung des beispielhaft analysierten Journals innerhalb der disziplinären Zeitschriftenlandschaft eingegangen. Im Anschluss werden computerlinguistische Verfahren vorgestellt. Die Darstellung des methodischen Vorgehens und die Präsentation der so zu erzielenden Ergebnisse wird am Beispiel der 2001 eingeführten bwp@ vorgenommen. Mithin stehen weniger die inhaltlichen Ergebnisse als vielmehr die methodischen Potenziale im Fokus. Der Beitrag schließt mit einer zusammenfassenden Diskussion und einem Ausblick auf weiterführende Arbeiten sowie weitere potenzielle Anwendungsfelder.

\section{2 (Berufs- und wirtschaftspädagogische) Wissenschaftsforschung und die Bedeutung von Fachzeitschriften}

Um die historische und aktuelle Verfassung bzw. Entwicklung wissenschaftlicher Disziplinen zu untersuchen, eignen sich nach Weingart und Winterhager (1984) verschiedene Merkmale, Elemente oder Dimensionen. So kann die Entwicklung wissenschaftlicher Disziplinen u.a. mithilfe outputorientierter Ansätze der empirischen Wissenschaftsforschung (z.B. Publikationsanalysen) untersucht werden. Als „sozial-kommunikative Netzwerke der Erkenntnisproduktion“ (Schriewer und Keiner 1993, S. 280) etablieren Disziplinen institutionsunabhängige und selbstverwaltete Kommunikationsformen: bspw. in Form wissenschaftlicher Gesellschaften, Kon-

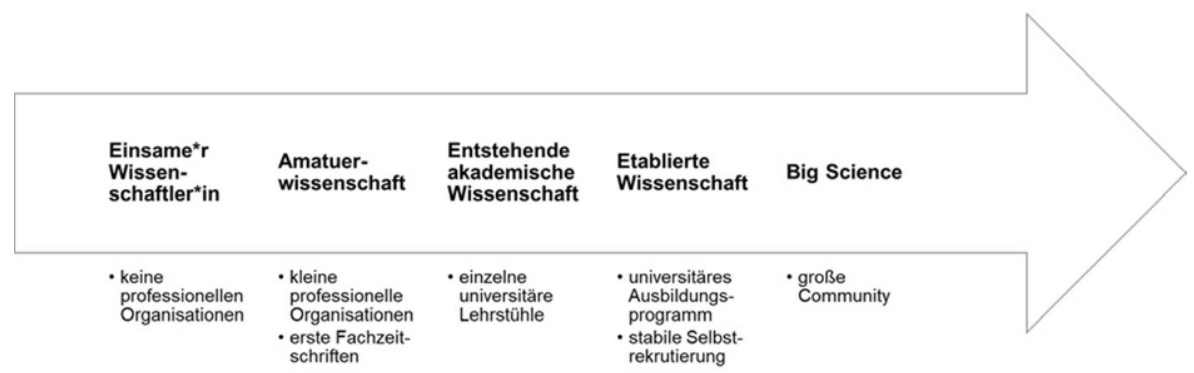

Abb. 1 Modell des Institutionalisierungsprozesses wissenschaftlicher Disziplinen (eigene Darstellung auf der Grundlage von Clark 1974, S. $109 \mathrm{ff}$. 
gressen und Tagungen oder eines selbstverwalteten Publikationssystems (Schriewer und Keiner 1993, S. 281). Auf Basis des Entwicklungs- bzw. Expansionsmodells wissenschaftlicher Disziplinen von Clark (1972, 1974) wird deutlich, dass Kommunikation bzw. die Etablierung, Professionalisierung und Ausweitung der Kommunikation(sbeziehungen) Übergänge zwischen verschiedenen Entwicklungsstadien markieren (Abb. 1).

Nach diesem Modell beginnt die Entwicklung einer neuen Disziplin mit einem*r einsamen Wissenschaftler*in, der*die neue Ideen kommuniziert bzw. publiziert und dadurch potenziell Anhänger*innen findet, die bereit sind, Ressourcen in die Weiterentwicklung der Idee und die Weiterführung der Kommunikation zu investieren. Damit erreicht die neue Idee das zweite Stadium der Amateurwissenschaft, in der erste kleine professionelle Organisationen (,scientific societies“) konstituiert und Fachzeitschriften etabliert werden. Wissenschaftliche Gesellschaften ermöglichen ihren Mitgliedern, regelmäßig zusammenzukommen, Präsentationen und Diskussionen über Beiträge zu ermöglichen und diese zu veröffentlichen (Clark 1974, S. 109 ff.; Götzl et al. 2018; Reinisch 2010, S. 189). ${ }^{1}$ Das dritte Stadium, die ,entstehende akademische Wissenschaft", beginnt mit der Einrichtung einzelner Lehrstühle an Hochschulen resp. Universitäten. Diesen Status erreichte die Wirtschaftspädagogik zwischen 1906 (mit Carl Theodor Dunker in Berlin), 1923 (mit Karl von der Aa in Leipzig) und 1930 (mit Friedrich Feld in Berlin) (Reinisch 2009; Götzl et al. 2018).

Wenn es den Inhaber*innen dieser Lehrstühle gelingt, ein Ausbildungsprogramm zu etablieren, für eine stabile Selbstrekrutierung aus dem eigenen wissenschaftlichen Nachwuchs zu sorgen und in der Folge die quantitative Ausweitung der Disziplin zu forcieren, ist das Stadium der etablierten Wissenschaft erreicht (Clark 1974, S. 110 ff.). Diesen Status spricht Reinisch (2009) der Berufs- und Wirtschaftspädagogik heute zu. Götzl et al. (2018) können diese Einschätzung vor dem Hintergrund kollektivbiografischer Daten empirisch stützen: Spätestens ab 1985, kann die Wirtschaftspädagogik als etablierte Wissenschaft bezeichnet werden. Das fünfte und letzte Stadium der Big Science ist vorläufig auszublenden, da nicht anzunehmen ist, dass die Berufs- und Wirtschaftspädagogik diesen Status in absehbarer Zeit erreichen wird. Zwar gibt es Aktivitäten der disziplinären Internationalisierung wie auch Prozesse der Differenzierung, Spezialisierung und Dezentralisierung, allerdings führt dies noch nicht zu einer weitgehenden Anonymisierung von Kommunikation und Aufstieg.

Kommunikation in Fachzeitschriften ist aber nicht nur als Ausgangspunkt für die Entwicklung wissenschaftlicher Disziplinen relevant. Sie bildet zugleich einen Ausgangspunkt im Sedimentationsprozess disziplinären Wissens. Papenkort (2015, S. $15 \mathrm{ff}$.) ordnet unterschiedliche Publikationsformen u.a. verschiedenen wissen-

\footnotetext{
1 Für die Berufs- und Wirtschaftspädagogik kann dieses Stadium für das Ende des 19. Jh. markiert werden. 1887 begann Oskar Pache (1843-1906) zusammen mit weiteren Schulmännern die Zeitschrift ,Die Fortbildungsschule“ (als Vorläufer der heutigen ZBW) herauszugeben. Diese trug wenig später den Untertitel „Centralorgan für das nationale Fortbildungsschulwesen“. Sie hatte zum Ziel, die Fortbildungsschulidee zu verbreiten. Pache war zugleich an der Gründung des ,Verbandes der Freunde und Lehrer deutscher Fortbildungsschulen“ (1892) beteiligt. Anfangs stand jedoch weitgehend die Praxis in der neuen Schulart im Mittelpunkt des Interesses (Klusmeyer 2001, S. 73 f.).
} 


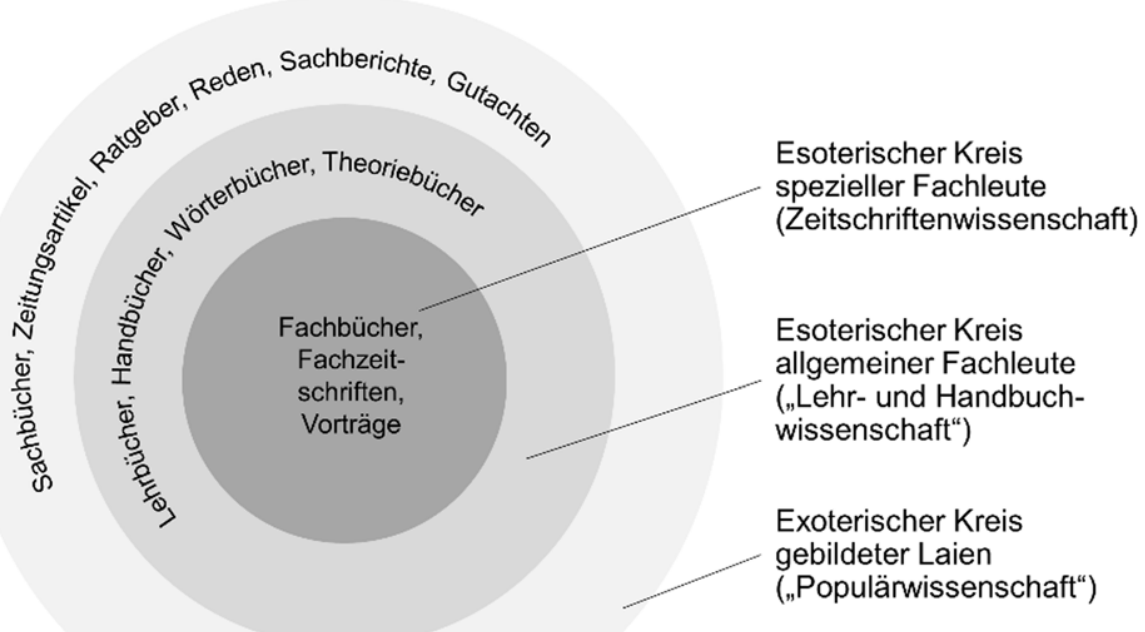

Abb. 2 Wissenschaftliche Publikationsformen (eigene Darstellung auf der Grundlage von Papenkort 2015, S. 16)

schaftlichen Kreisen zu. Dies erfolgt unter Rückgriff auf die Arbeiten von Fleck ([1935] 1980) sowie von Krohn und Küppers (1989). Fleck (1980, S. 147f.) unterscheidet drei „Kreise“ der Wissenschaft, denen unterschiedliche Publikationsformen zuordnet werden (Abb. 2). Im Zentrum einer Disziplin stehen spezielle Fachleute, die grundlegende Forschungsergebnisse generieren und v. a. in Fachzeitschriften mit anderen Spezialist*innen kommunikativ teilen (,Zeitschriftenwissenschaft“). Daneben befindet sich der Kreis allgemeiner Fachleute, die als „Lehrbuch-“ bzw. „Handbuchwissenschaft" der disziplinären Orientierung dienen und zur Rekrutierung von qualifizierten Nachwuchs beitragen. Außen erfolgt dann populärwissenschaftliche Kommunikation mit der ,Praxis'.

Publikationen, die in Fachzeitschriften gedruckt und somit vielen interessierten Rezipienten*innen zugänglich gemacht werden, sind damit ein wichtiges Instrument disziplinspezifischer Kommunikation. Die Form einer Fachzeitschrift „erweist sich in ungewöhnlichem Maße als sowohl instrumentell wie auch symbolisch adäquat für die Probleme der Kommunikation in disziplinären Gemeinschaften" (Stichweh 1984, S. 425). Durch die kontinuierliche Veröffentlichung und den durch Publikation stets wachsenden Bestand an Fachartikeln symbolisieren Fachzeitschriften eine themenund arbeitsbereichsbezogene Kollektivität, Beständigkeit sowie Unbegrenztheit des wissenschaftlichen Diskurses. Ihre Offenheit steht für die fluide Gemeinschaft derer, die Interesse an der jeweiligen wissenschaftlichen Disziplin haben (Klusmeyer 2001, S. 30 ff.). Sie ist geprägt von Diskursivität, d.h. die Kommunikation geht prinzipiell über eine einseitige Sender-Empfänger-Kommunikation hinaus (Stichweh 1984, S. 427). Damit erlauben Fachzeitschriften nicht nur einen Einblick in die kognitive Verfassung einer Disziplin, d.h. welche Themen, Problem- bzw. Frage- 
Tab. 1 Bedeutung disziplinärer Fachzeitschriften (Auszug) [Daten: Klusmeyer et al. 2011; Söll et al. 2014]

\begin{tabular}{|c|c|c|c|c|c|}
\hline & $\begin{array}{l}\text { (Vorstudie) } \\
\text { Publikations- } \\
\text { aufkommen } \\
\text { (Rang) }\end{array}$ & $\begin{array}{l}\text { Reputation } \\
\text { (Mittelwert) }\end{array}$ & $\begin{array}{l}\text { Reputation } \\
\text { (Rang) }\end{array}$ & $\begin{array}{l}\text { Relevanz } \\
\text { (Mittelwert) }\end{array}$ & $\begin{array}{l}\text { Relevanz } \\
\text { (Rang) }\end{array}$ \\
\hline $\begin{array}{l}\text { Zeitschrift für Berufs- und } \\
\text { Wirtschaftspädagogik (ZBW) }\end{array}$ & 1 & 4,96 & 3 & 4,93 & 1 \\
\hline Berufsbildung & 2 & 3,08 & 17 & 3,62 & 11 \\
\hline $\begin{array}{l}\text { Berufsbildung in Wissenschaft } \\
\text { und Praxis (BWP) }\end{array}$ & 3 & 3,48 & 15 & 3,96 & 7 \\
\hline Die berufsbildende Schule & 4 & 2,97 & 20 & 3,58 & 13 \\
\hline Grundlagen der Weiterbildung & 5 & 2,80 & 21 & 2,91 & 20 \\
\hline Wirtschaft- und Erziehung & 6 & 2,98 & 19 & 3,55 & 15 \\
\hline $\begin{array}{l}\text { Berufs- und Wirtschaftspäd- } \\
\text { agogik - online (bwp@) }\end{array}$ & $(-)$ & 4,27 & 8 & 4,86 & 2 \\
\hline
\end{tabular}

Anm.: Aus den beiden Lehrer*innenverbandszeitschriften „Die berufsbildende Schule“ sowie „Wirtschaft und Erziehung“ ist mit der Fusion der Verbände im Jahr 2018 die Zeitschrift „Bildung und Beruf“ hervorgegangen

stellungen mit welchen Methoden bearbeitet bzw. verhandelt werden (Ideengestalt), sondern auch wer mit wem (wie) kommuniziert bzw. wer Teil der wissenschaftlichen Gemeinschaft ist (Klusmeyer 2001) oder einer ihrer Schulen, die sich durch eine gemeinsame paradigmatische Orientierung auszeichnen (Kauder 2010).

Für die Berufs- und Wirtschaftspädagogik stellt Reinisch fest, dass (Fach-)Zeitschriften in Vergangenheit und Gegenwart ,(...) die zentralen Medien des wissenschaftlichen und politischen Diskurses“ (Reinisch 2006, S. 159) sind. Neben (internationalen) erziehungswissenschaftlichen Zeitschriften nutzt sie vor allem einige ,wenige“ disziplinspezifische Fachzeitschriften (Tab. 1). Daneben kommuniziert man ebenfalls stark über Monografien und Sammelwerke (Söll et al. 2014), was jedoch nach Papenkort (2015) ebenso Ausdrucksformen einer ,Zeitschriftenwissenschaft" sind. Bedeutsamste Zeitschrift ist die o.g. ZBW im Hinblick auf Reputation und Relevanz (Söll et al. 2014)2.

Klusmeyer et al. (2011) sowie Söll et al. (2014) zeigen aber auch, dass die Berufs- und Wirtschaftspädagogik - online (bwp@) - die aus forschungsmethodischen Gründen im Rahmen der Erprobung des Verfahrens beispielhaft genutzt wird - hinter der angesprochenen ZBW die höchste Relevanz für die Disziplin und hinter weiteren erziehungswissenschaftlichen Journals (z.B. „Zeitschrift für Pädagogik“ und ,Zeitschrift für Erziehungswissenschaft“) die zweithöchste Reputation der dis-

\footnotetext{
2 Söll et al. veröffentlichten 2014 eine Studie über das „Rezeptions- und Publikationsverhalten“ innerhalb der Berufs- und Wirtschaftspädagogik. Sie befragten die Mitglieder der Sektion Berufs- und Wirtschaftspädagogik erstens nach bedeutsamen Zeitschriften. Hier wurden - neben den in Tab. 1 aufgeführten disziplinären Zeitschriften - erziehungswissenschaftliche Zeitschriften (z. B. Zeitschrift für Erziehungswissenschaft, Zeitschrift für Pädagogik) sowie internationale Journale genannt. Die 25 am häufigsten genannten Zeitschriften waren dann in einer zweiten Befragung hinsichtlich ihrer Relevanz (für die disziplinäre Kommunikation) und Reputation (i. S. reputierlicher Bedeutung einer Veröffentlichung für die akademische Karriere) zu bewerten (siehe ausführlich Söll et al. 2014, S. 508 ff.).
} 
ziplinären Zeitschriften besitzt. Somit - so konstatieren Söll et al. (2014, S. 516) mit Verweis auf Taubert und Weingart (2010, S. 172) - lassen sich die aus anderen Domänen bekannten „Zentrum-Peripherie-Strukturen“ auch in der berufs- und wirtschaftspädagogischen Zeitschriftenlandschaft wiederfinden, in der eine relativ große Zahl weniger renommierter Zeitschriften eine kleine Zahl angesehener Journale umgibt. ${ }^{3}$

Diese bwp@ (ISSN: 1618-8543) soll im Folgenden kurz vorgestellt werden. Sie erscheint i.d.R. zweimal jährlich als Online-Journal. Nach eigenen Angaben ist sie seit 19.11.2001 online. Es haben bislang ca. 1220 Autor*innen mehr als 1200 Beiträge veröffentlicht (https://www.bwpat.de/ueber-bwp; 20.08.2020). Zielgruppen sind „Berufs- und WirtschaftspädagogInnen an den Universitäten in Lehre, Forschung und im Studium, in der Schule, in der betrieblichen und institutionellen Aus- und Weiterbildung und in der Bildungsverwaltung“ (ebd.). Sie versteht sich als Fachzeitschrift, ,,in der Beiträge aus Theorie und Praxis der Berufs- und Wirtschaftspädagogik publiziert werden [und; d. V.] die aktuelle Schwerpunktthemen aufgreift und aus unterschiedlichen Perspektiven präsentiert und zur Diskussion anbietet" (Büchter und Gramlinger 2001). Als unabhängiges Medium hat sie sich dem „open content Prinzip“ verpflichtet (Büchter et al. 2011). Die bwp@ hat sich in ihren ersten nunmehr fast 20 Jahren eine zentrale Stellung in der disziplinären Zeitschriftenlandschaft gesichert (Söll et al. 2014). Dies mag aus Sicht der Autoren*innen am zügigen Publikationsverfahren, aus Sicht der Rezipienten*innen an dem leichten, kostenfreien Zugriff auf die Beiträge liegen. Inwieweit ihre Etablierung auch als Ausdruck wissenschaftlicher Ökonomisierung und eines zunehmenden Publikationsdrucks zu interpretieren ist, durch den ,die Zahl der Publikationen bzw. die Länge der Publikationsliste vor der Qualität der Beiträge rangiert“ (Büchter et al. 2011, S. 2) diskutieren ihre Herausgeber*innen selbst kritisch.

Die bwp@ erscheint in verschiedenen Formaten. Im Zentrum der Zeitschrift stehen die zweimal jährlich erscheinenden regulären Themenausgaben. In diesen mittlerweile 36 Ausgaben werden Beiträge mit einem je unterschiedlichen thematischen Fokus publiziert (Tab. 2) ${ }^{4}$. Mittels Call for Papers wird die Scientific Community aufgerufen, entsprechende Beiträge einzureichen, die einem herausgeber*innenbasierten double-blind Review-Verfahren unterzogen werden (Büchter et al. 2011, S. 5 f.).

Diese regulären Themenausgaben werden im Weiteren für die Analyse genutzt, da sie sich für den methodischen Zweck der Analyse - einer Validierung des genutzten Topic-Modeling-Ansatzes - am besten eignen. Daneben besteht ein Format „bwp@-Profile“, das unregelmäßig erscheinende, digitale Festschriften enthält, sowie ein Format „bwp@-Spezial“, dessen 15 Ausgaben ein breites Themenspektrum

\footnotetext{
3 Ca. 40,3\% des Publikationsaufkommens der Disziplin sind Fachzeitschriften zuzuordnen, sodass die Mitglieder der Sektion Berufs- und Wirtschaftspädagogik innerhalb der DGfE - wie im Übrigen auch andere geistes- und sozialwissenschaftliche Disziplinen - auch stark in Sammelwerken und Monografien publizieren (Söll et al. 2014). Zu einem ähnlichen Bild kommt Baumann (2009) für die österreichische Berufs- und Wirtschaftspädagogik.

$4 \mathrm{Zu}$ erwähnen ist, dass es für jede Ausgabe nicht nur einen Veröffentlichungstermin gibt, sondern dass bereits veröffentlichte Ausgaben z. T. in mehreren Tranchen um weitere Beiträge ergänzt werden.
} 
Tab. 2 Themen der regulären Ausgaben (Auszug) [Daten: https://www.bwpat.de/alle, 20.08.2020]

\begin{tabular}{llll}
\hline $\begin{array}{l}\text { Aus- } \\
\text { gabe }\end{array}$ & Jahr & Titel & $\begin{array}{c}\text { Anzahl } \\
\text { Artikel }\end{array}$ \\
\hline 1 & 2001 & (K)ein Ende der Diskussion um die Reform der Berufsschullehrerausbildung? & 5 \\
2 & 2002 & Lernen in Netzen - Aufgaben für die Berufs- und Wirtschaftspädagogik & 8 \\
3 & 2002 & $\begin{array}{l}\text { Kooperation in der beruflichen Aus- und Weiterbildung - Anspruch und Rea- } \\
\text { lität einer Reformbedingung }\end{array}$ & 9 \\
4 & 2003 & Lernfeldansatz zwischen Feiertagsdidaktik und Alltagstauglichkeit & 22 \\
5 & 2003 & Regionale Berufsbildungszentren & 12 \\
6 & 2004 & Förderung benachteiligter und behinderter Jugendlicher & 18 \\
7 & 2004 & Vocational and Business Education and Training in Europe: Qualifications and & 18 \\
& & the World of Work & 22 \\
$(\ldots)$ & & & Inklusion in der beruflichen Bildung \\
30 & 2016 & Entwicklungsperspektiven des beruflichen Schulwesens. Institutionen, Steue- \\
31 & 2016 & 13 \\
32 & 2017 & Betrieblich-berufliche Bildung & 21 \\
33 & 2017 & Entwicklungsbezogene (Praxis-)Forschung & 21 \\
34 & 2018 & Was berufliche und akademische Bildung trennt und verbindet & 23 \\
36 & 2018 & Ökonomisierung in der Bildung und ökonomische Bildung & 15
\end{tabular}

haben und u. a. zur z. T. sehr umfangreichen Tagungsdokumentation dienen. Weiterhin erschienen seit 2001 regelmäßig Rezensionen sowie bislang 218 Newsletter.

\section{Fragestellungen und Methodik}

\subsection{Fragestellungen}

Die Fragestellung dieses Beitrages fokussiert ein methodisches Problem bei der inhaltlichen Analyse von Fachzeitschriften. Es hat sich am Beispiel empirischer Analysen der o.g. ZBW (Jahn et al. 2019; Klusmeyer 2001; Tenberg et al. 2009) gezeigt, dass traditionelle inhaltsanalytische Verfahren mit verschiedenen Problemen zu kämpfen haben. Klusmeyer (2001) bspw. analysierte deren Beiträge im Rahmen einer Teilerhebung $(n=1192)$ im Zeitraum zwischen 1960 und 1998 im Hinblick auf ihre formale, soziale und kognitive Dimension. Die formale Dimension bezieht sich auf Daten zu den publizistischen Einheiten selbst (z. B. Titel, Jahr, Seitenzahl), die soziale auf Angaben zu den Autor*innen (z. B. Name(n), Anzahl der Verfasser*innen, Geschlecht, Position, Institution) und die kognitive auf die in den Beiträgen kommunizierten Wissenselemente, wie verhandelte Themen, angewandte Methoden, intendierte Aussage- und Wissensstrukturen. Die thematische Codierung der Beiträge erfolgte über eine quantitativ ausgerichtete Inhaltsanalyse. Die Beiträge wurden erstens vollständig gelesen und zweitens mithilfe des deduktiv ausgerichteten Kategoriensystems summarisch codiert (Klusmeyer 2001, S. $82 \mathrm{ff}$., S. $105 \mathrm{ff}$. u. S. 305 ff. sowie insb. Hentke 1982; Macke 1989). 
Im Kontrast zu dem hier vorgestellten induktiven-computerlinguistischen Verfahren, nutzte Klusmeyer einen deduktiven Zugriff. Das gewählte Kategoriensystem ist aber nur als Kompromiss verschiedener disziplinärer Kartierungsansätze zu verstehen (Klusmeyer 2001, S. $89 \mathrm{ff}$.). Es ist fraglich, ob solche ,älteren' thematischen Systematisierungsversuche heutige und zukünftige Themen assimilieren können. Es erscheint vielmehr notwendig, dass diese mit den Entwicklungen fragmentierter Disziplinen (Ambrose et al. 2010) schritthalten. Problematisch ist ferner der Ansatz, die einzelnen Beiträge summarisch im Bestreben zu codieren, eindeutige Zuordnungen (Einfachcodierungen) vorzunehmen. Damit werden weitere, nachgelagerte, trotzdem relevante Themen innerhalb der Artikel ausgeblendet (Jahn et al. 2019). Letztlich kann auch nicht ausgeschlossen werden, dass Mitglieder der Disziplin in anderen Fachzeitschriften über andere Themen in anderer Art und Weise kommunizieren bzw. dass bestimmte Mitglieder oder Gruppen der Disziplin gar nicht oder nicht dominant in der ZBW bzw. überhaupt in Fachzeitschriften publizieren. Daher wäre eine induktive Analyse der gesamten disziplinären Kommunikation vorzunehmen, um ein valides Bild der disziplinären Kommunikation (zumindest in den esoterischen Kreisen) zeichnen zu können. Dies lässt sich allerdings kaum mit traditionellen inhaltsanalytischen Verfahren realisieren.

Vor diesem Hintergrund stellt sich die Frage, ob sich maschinelle, computerlinguistische Verfahren zur Analyse der disziplinären Kommunikation eignen, die angesprochenen Probleme zu lösen. Mit ihnen ist erstens die Verarbeitung großer Datenmengen aller Artikel (einer oder auch mehrerer Zeitschriften sowie anderer Publikationsformen) möglich. Zweitens ermöglichen sie reproduzierbare (annähernd rein) induktive Codierungen und eine inhaltlich-thematische Kartierung disziplinärer Kommunikation. Eine ex-ante Kategorisierung disziplinärer Themen und Inhalte ist damit nicht mehr notwendig. Drittens erlauben sie eine Quantifizierung der Themendominanz in einzelnen Analyseeinheiten (Artikeln) und somit objektiv gewichtete Mehrfachcodierungen. Ob das computerlinguistische Verfahren dies leisten kann, sollim Weiteren am Beispiel der bwp@ untersucht werden.

Unterhalb dieses eher methodisch orientierten Problems liegt die inhaltliche Fragestellung des Beitrages: Wer (soziale Dimension) kommuniziert über welche Themen (kognitive Dimension) in der bwp@? Die Fragestellung orientiert sich an der Forschungslogik der angesprochenen Studie von Klusmeyer (2001). Vor diesem Hintergrund ergeben sich folgende Teilfragen:

\section{Soziale Dimension:}

- Wie entwickelt sich die Autoren*innenschaft hinsichtlich ihrer institutionellen Zugehörigkeit (Affiliation)?

- Wie entwickelt sich die Zeitschrift im Hinblick auf die Offenheit für Autoren*innen bzw. liegt ein starker Einfluss weniger Autor*innen vor?

- Wie entwickelt sich die durchschnittliche Anzahl der Beiträge mit Autoren*innenkooperationen pro Jahrgang? 


\section{Kognitive Dimension:}

- Welche Themen werden in der Zeitschrift be- bzw. verhandelt?

- Welche Themen werden wie stark in einzelnen Beiträgen be- bzw. verhandelt?

- Inwieweit korrespondieren die Ergebnisse des (computerlinguistischen) TopicModeling-Verfahrens mit den Themen von Artikeln und Heften?

\section{Sozial-kognitive Dimension}

- Welche Autoren*innen bzw. Institutionen be- bzw. verhandeln welche Themen?

- Welche Ähnlichkeiten lassen sich identifizieren?

\section{2 Überlegungen zum methodischen Vorgehen}

Die wissenschaftstheoretisch inspirierte Analyse von Fachzeitschriften mit Hilfe von computerlinguistischen Verfahren ist nicht völlig neu (Cretchley et al. 2010; Liesch et al. 2011; Lin und Lee 2012; Zawacki-Richter und Naidu 2016). Die zitierten Arbeiten nutzten das Programm leximancer ${ }^{\mathrm{TM}}$, dessen Quellcode und Algorithmus allerdings nicht zugänglich ist. Daher wird ein Verfahren vorgestellt, das auf dem LDA Algorithmus von David Blei (2012) basiert. Computerlinguistik nutzt grundlegende Ergebnisse des maschinellen Lernens, welches in einer überwachten und unüberwachten Form existiert (Goldenstein et al. 2019). Überwachtes Maschinelles Lernen bedarf stets eines bereits existierenden Kategoriensystems. Das bedeutet, dass bspw. das Kategoriensystem von Klusmeyer für eine computerlinguistische Analyse auf Basis überwachten maschinellen Lernens verwendet werden könnte. Maschinelles Lernen ermöglicht es auf Basis der vorgegebenen Kategorien und der diesen zugeordneten Textstellen computergestützt diejenigen sprachlichen Muster zu identifizieren, welche die Kategorien voneinander trennen. Das Ergebnis dieses sogenannten Trainings ist ein maschinell erzeugtes Modell, welches genutzt werden kann, um das Auftreten der trainierten Kategorien in unbekannten Texten vorherzusagen. In Summe kann überwachtes maschinelles Lernen die Analyse großer Textmengen auf Basis ex-ante definierter Kategorien erheblich erleichtern (Kobayashi et al. 2018). Allerdings ermöglicht überwachtes maschinelles Lernen somit nicht, über bereits existierendes Wissen hinauszugehen. Hierzu bedarf es der induktiven Erzeugung von Kategorien.

Unüberwachtes maschinelles Lernen in der Computerlinguistik will bislang unbekannte Muster aus Sprachdaten extrahieren. Da in diesem Aufsatz diese Analysestrategie - im Kontrast zum traditionellen deduktiven Ansatz - als sinnvoll erscheint, wurde ein Topic Modeling durchgeführt. Im Gegensatz zu Methoden, die auf überwachtem maschinellem Lernen basieren, stellt Topic Modeling eine Form sog. generativ-probabilistischer Modelle dar, die keine ex-ante definierten Kategorien benötigen (Blei 2012). Stattdessen identifiziert Topic Modeling Kategorien in Texten auf Basis latenter semantischer Strukturen (als Topics), indem statistisch auffällige Muster von Wortkombinationen aufdeckt werden (Goldenstein und Poschmann 2019a, b; Hannigan et al. 2019; Mohr und Bogdanov 2013). So ergibt sich die thematische Semantik bspw. von Wörtern wie „Inklusion“, „Behinderung“, „Integration“ und „Teilhabe“ aus ihrer wiederkehrenden relativen Nähe zueinander in 
Texten (s. unten). Arbeiten, die sich mit der Evaluation der Ergebnisse von Topic Modeling im Vergleich zu händischen Kodierungen befassen, zeigen, dass diese Technik hervorragend geeignet ist, um automatisiert semantische Strukturen in großen Textmengen zu identifizieren (Muller et al. 2016; Nelson et al. 2018). In den maschinellen Prozess wird nur ex-ante durch die Auswahl und Aufbereitung der Daten, durch die Verwendung von Stopp-Listen zur Eliminierung häufig auftretender und gewöhnlich irrelevanter Wörter (z.B. ,und“, ,oder“) sowie durch die Definition der Anzahl an Themen eingegriffen. Die Anwendung von Topic Modeling erfordert es demnach, die Anzahl der Themen, d.h. der zu entdeckenden latenten semantischen Strukturen, festzulegen. Es ist deshalb ratsam, mehrere Konfigurationen zu testen und anhand mathematischer Operationen, wie z. B. der log-likelihood Berechnung, die optimale Spanne an Themen zu ermitteln. Allerdings ist es stets ratsam die Anzahl der Themen aus dieser Spanne so zu wählen, dass diese interpretierbar und analytisch nützlich sind. Insofern enthebt auch ein Topic Modeling Forschende nicht davon, eine externe Validierung durchzuführen (DiMaggio et al. 2013; Goldenstein und Poschmann 2019a, b; Nelson 2017).

Im Hinblick auf die fokussierte Fragestellung und das Datenmaterial hat dieser Ansatz erhebliche Vorteile. Er ermöglicht nicht nur die Verarbeitung (Volltextanalyse) der gesamten Artikel einer Zeitschrift (oder mehr) sowie eine induktive Themenanalyse. Er ist auch ein weitgehend objektives Verfahren, das reproduzierbare Ergebnisse erzeugt. Zudem erlaubt der Ansatz die Quantifizierung der Themendominanz in einzelnen Beiträgen, d.h. eine objektiv gewichtete Mehrfachcodierung. Dieser Aspekt ist eine substanzielle methodische Weiterentwicklung für die Analyse der disziplinären Kommunikation. Ein Nachteil des Verfahrens besteht darin, dass das so errechnete Themenmodell zumindest zunächst primär für den analysierten Gegenstand gilt. Dessen Übertragbarkeit auf andere Gegenstände - in unserem Fall disziplinäre Fachzeitschriften oder die gesamte disziplinäre Kommunikation - ist abhängig von der Repräsentativität des analysierten Artefaktes.

\subsection{Darstellung von Datenerhebung und -auswertung}

Im Folgenden wird die Datenerhebung und -auswertung mittels des eben beschriebenen Verfahrens erläutert. Im ersten Schritt wurden alle 630 bwp@-Artikel der Regelausgaben (Ausgabe 1-34) heruntergeladen, exklusive der jeweils einführenden Editorials. Anschließend wurden 20 englischsprachige Beiträge ausgeschlossen, da für die Analyse kein Mehrwert entsteht, wenn englische Beiträge allein aufgrund einer anderen Sprache einem eigenen Topic zugewiesen würden. Insgesamt bestand der Datenkorpus dann nur noch aus 610 Artikeln der Jahre 2001-2018. Im zweiten Schritt (Abb. 3) wurden Daten zur sozialen Dimensionen (Autoren*innenname, institutionelle Zugehörigkeit bzw. Affiliation, Anzahl) extrahiert und standardisiert. Die Affiliationen wurden Arbeitsbereichen (z.B. Universität, Forschungsinstitute, Schulpraxis, etc.) zugeordnet.

Im dritten Schritt wurde der Datenkorpus der computerlinguistischen Analyse unterzogen. Im Ergebnis wurden verschieden stark differenzierte Topic-Listen (10er, 20er, 30er, 40er, 100er Topic-Liste) generiert. Je weniger differenziert die Listen sind, umso trennschärfer aber auch umso abstrakter sind die Themen. Je umfang- 


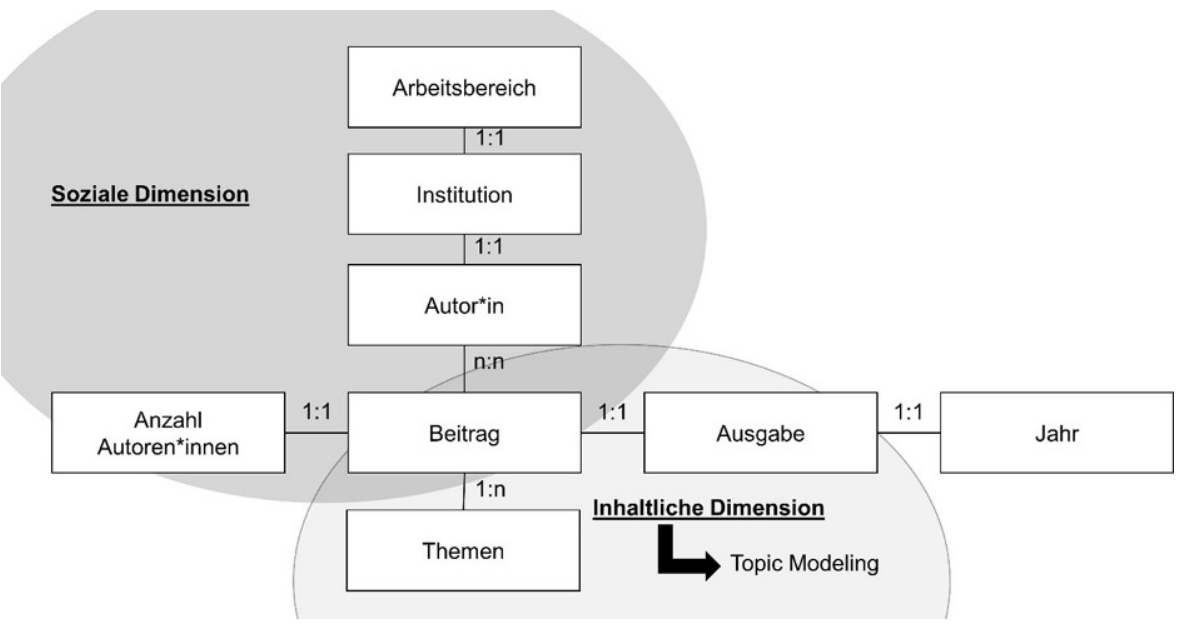

Abb. 3 Vereinfachte Datenstruktur

reicher die Listen, umso differenzierter aber weniger trennscharf sind sie. Die identifizierten Topics sind Wortlisten, die schließlich interpretiert und mit einem Label versehen werden müssen (Abb. 4). Bemerkenswert ist, dass die Wortlisten Hinweise auf potenzielle Protagonisten*innen (durch entsprechende Zitationen) enthalten (nicht zu verwechseln mit den Autoren*innen).

Im vierten Schritt wurden die Themenkonfigurationen (Topic-Listen) gesichtet und die Variante mit 40 Topics ausgewählt, da sie erstens differenziert genug ist und sie zweitens noch einen trennscharfen Überblick ermöglicht. Anschließend wurden 6 Themen für weitere Analysen ausgeschlossen, da sie entweder englischsprachig waren oder sich auf Füllwörter bzw. Formulierungen aus Abstracts oder Einleitungen bezogen. Für die weiteren Analysen ist es nützlich, die 34 verbliebenen Topics mit einer Überschrift zu versehen. Dies ist ein interpretativer Prozess. Das Bilden der Labels (Überschriften der Topics) und deren Validierung erfolgt in einer In-
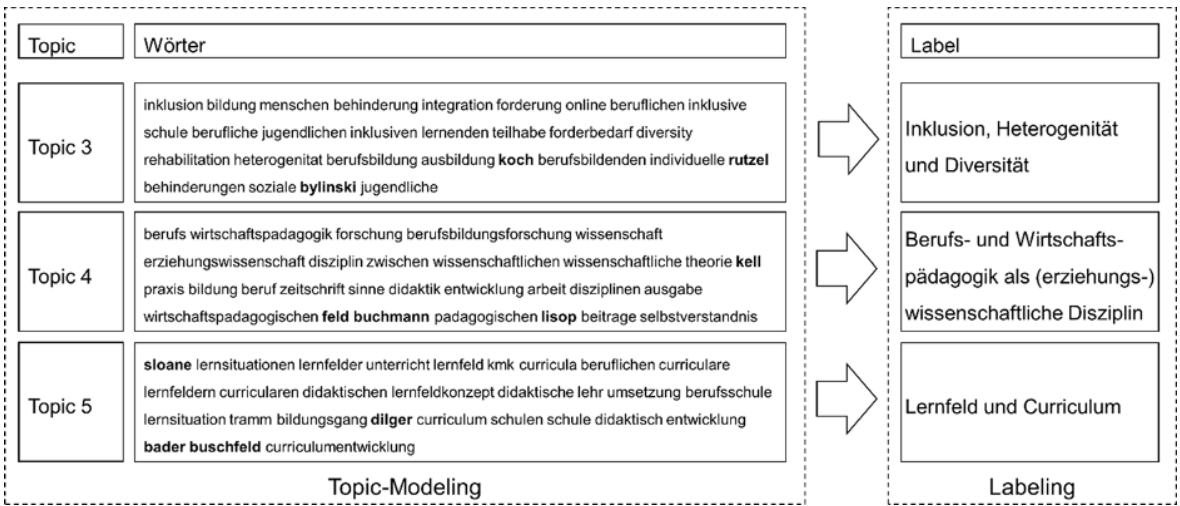

Abb. 4 Wortlisten und Labels (Beispiele) 
terpretationsgruppe $(\mathrm{N}=5)$. Das Labeling ist vor allem für die Interpretation der Themenstrukturen und -konjunkturen sinnvoll. Für die Analyse paradigmatischer Entwicklungen, von Ähnlichkeiten und Unterschieden sowie von Prozessen der Angleichung und Differenzierung sind die Formulierungen der Labels im Sinne einer Überschrift weniger bedeutsam als vielmehr die zugrunde liegenden Modelle. Die Formulierung der Labels erfolgt mehrstufig. Zunächst wurden je Topic die Wortlisten (Abb. 4) der zentralen, repräsentativen Begriffe gesichtet, in ihren Bezügen zueinander diskutiert und kontextualisiert. Auf dieser Basis können diskursiv erste Versionen von Labels erarbeitet und vorläufig formuliert werden. Anschließend wurden Beiträge, in denen die Topics mit relativ starker Gewichtung vorkommen, gelesen. Anschließend wurden die Labels teilweise reformuliert und dabei weiter oder enger gefasst. Dieses interpretative Labeling erfolgt somit zwar intersubjektiv, allerdings nicht objektiv und es ist nicht auszuschließen, dass andere Gruppen zu abweichenden Formulierungen gelangen.

Im fünften Schritt wurden die Topics validiert. Da jeder Beitrag aus mehreren Themen besteht, wurden für jedes zu validierende Topic die fünf Beiträge extrahiert, die es am stärksten enthalten (i.d.R. zu min. 40\%). Diese Beiträge wurden durch die Mitglieder der Interpretationsgruppe gelesen. Dabei wurde geprüft, ob diese Beiträge das jeweilige Thema erkennbar enthalten. Das bedeutet auch, dass starke Topics in Beiträgen nicht automatisch den inhaltlichen Schwerpunkt eines Beitrags markieren müssen, was im Besonderen für methodische oder methodologische Topics (wie z. B. ,Design-Based-Research“) gilt.

\section{Darstellung und Diskussion der Einzelbefunde}

\subsection{Analyse der sozialen Gestalt}

Im Hinblick auf die soziale Dimension wurden die Namen der Autoren*innen und ihre institutionelle Herkunft (Affiliation) sowie die Anzahl der Autoren*innen pro Beitrag erfasst und ausgewertet. Zwischen Autoren*innen und Beiträgen besteht eine $n: n$-Relation (Abb. 3). Die Analyse der 610 Beiträge zeigt, dass diese von 1016 Autoren*innen zunehmend in Mehrautoren*innenschaft verfasst wurden und insgesamt 618 verschiedene Autoren*innen beteiligt waren. 70\% der Autoren*innen haben lediglich einen Beitrag publiziert, 187 Personen waren demnach an mehr als einem Beitrag beteiligt. Zudem existiert eine Gruppe, die relativ häufig Beiträge publizieren. Diese „Vielschreiber*innen“ (erste 12\% der Autoren*innen verfassen $30 \%$ der Artikel) sind jedoch nicht so dominant, um ihnen einen übermäßig starken Einfluss zuzuschreiben.

Etwas anders verhält es sich mit der institutionellen Herkunft der Autoren*innen (Abb. 5). Die ersten 5 von 203 verschiedenen Institutionen stellen $25 \%$ der Autoren*innen und die ersten 15 Institutionen 50\%. Dies mag mit Blick auf eine kleine Disziplin wenig überraschen, zumal größere Standorte (mit mehreren Professuren) besonders dominant sind. Problematisch ist der Umstand, dass bestimmte (insb. quantitativ empirisch ausgerichtete) Standorte kaum oder gar nicht vertreten sind (z.B. Konstanz, Mannheim, Frankfurt, Göttingen, Leipzig, Berlin, Dresden). Vor 


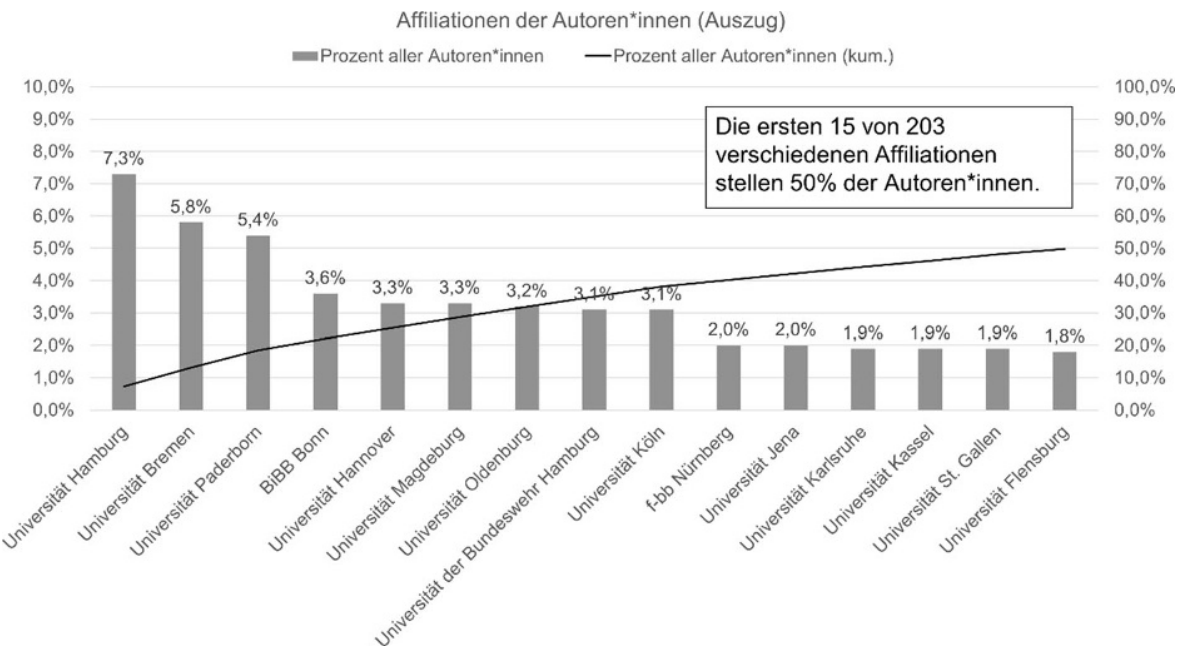

Abb. 5 Affiliationen der Autoren*innen (Auszug)

diesem Hintergrund wäre kritisch zu diskutieren, ob es der bwp@ hinreichend gelingt, die gesamte Disziplin zu repräsentieren und kommunikativ einzubinden oder sie „nur“ ein Kommunikationsmedium einer Schule, eines Denkkollektivs, eines Paradigmas ist. Ob die mangelnde Beteiligung bestimmter Mitglieder der Berufsund Wirtschaftspädagogik nun der geringeren Reputation (in Relation zu anderen (internationalen) Zeitschriften; Söll et al. 2014) oder der thematischen Ausrichtung der einzelnen Themenhefte geschuldet ist oder andere Ursachen hat, muss hier genauso offen bleiben, wie die Frage, ob die bwp@ einen substanziellen Beitrag zur diskursiven Verbindung der polarisierend unterschiedenen Grundlagen- und Anwendungsforschung (Büchter et al. 2011, S. 5) leisten konnte. Ferner kann der Blick auf die Arbeitsbereiche der Autoren*innen gerichtet werden. Autoren*innen aus dem Hochschulbereich dominieren in der Analyse mit etwa 79,1\%. Ebenso sind öffentliche und private Forschungsinstitute (insb. BiBB und f-bb) bedeutsame Akteure in der disziplinären Kommunikation $(10,1 \%)$. Autoren*innen aus der schulischen bzw. betrieblichen Praxis sind seltener beteiligt. Dies korrespondiert mit Ergebnissen zur Entwicklung der ZBW, wenngleich dort der Anteil von „Praktikern*innen“ nochmals geringer ist (Jahn et al. 2019; Klusmeyer 2001). Eine gleichberechtigte Kommunikation zwischen „Theorie“ und „Praxis“ findet in der bwp@ weniger statt, sodass sie vornehmlich eine Zeitschrift der wissenschaftlichen Berufs- und Wirtschaftspädagogik ist.

Tab. 3 zeigt, dass kooperative Publikationen seit Beginn der 2000er Jahre, auch standortübergreifend, deutlich zunehmen, was für die sozial-kognitive Dimensionen bedeutsam ist und mit einer generell kooperativeren Forschungs- und Publikationskultur erklärt werden kann (Jahn et al. 2019; Keiner 1999; Klusmeyer 2001). 
Tab. 3 Anzahl der Autoren*innen pro Beitrag

\begin{tabular}{llllllll}
\hline $\begin{array}{l}\text { Autoren* } \\
\text { innen }\end{array}$ & $2001-2003$ & $2004-2006$ & $2007-2009$ & $2010-2012$ & $2013-2015$ & $2016-2018$ & $2001-2018$ \\
\hline 1 & $66 \%$ & $72 \%$ & $44 \%$ & $47 \%$ & $50 \%$ & $38 \%$ & $\mathbf{5 2 \%}$ \\
2 & $25 \%$ & $22 \%$ & $42 \%$ & $29 \%$ & $33 \%$ & $37 \%$ & $\mathbf{3 2 \%}$ \\
3 & $7 \%$ & $5 \%$ & $8 \%$ & $15 \%$ & $11 \%$ & $15 \%$ & $\mathbf{1 0 \%}$ \\
$>3$ & $2 \%$ & $1 \%$ & $6 \%$ & $9 \%$ & $7 \%$ & $10 \%$ & $\mathbf{6 \%}$ \\
$N$ & 56 & 109 & 106 & 104 & 135 & 100 & $\mathbf{6 1 0}$ \\
MW & 1,45 & 1,34 & 1,79 & 1,93 & 1,78 & 2,07 & $\mathbf{1 , 7 5}$ \\
\hline
\end{tabular}

\subsection{Analyse der kognitiven Gestalt}

Die Erfassung der thematischen Ausrichtung der analysierten 610 Beiträge erfolgte mittels des vorgestellten computerlinguistischen Vorgehens. Insgesamt konnten 34 Topics identifiziert und interpretativ gelabelt werden (Abb. 6). Die disziplinäre Kommunikation in der bwp@ fokussiert vor allem auf ,originäre berufs- und wirtschaftspädagogische Gegenstandsfelder in Theorie und Praxis“, und damit jene Hauptkategorie, die in der Untersuchung von Klusmeyer (2001) auch die ZBW dominiert. Curriculare und didaktische Beiträge, Artikel zu Lehrenden und Lernenden sowie Beiträge, die strukturelle, konzeptionelle und organisationale Aspekte der Institutionen fokussieren, haben hohe Bedeutung. Wenn die Disziplin als Wissenschaft ,über die bildende Vorbereitung des Menschen zur Bewältigung von sozioökonomischen Lebenssituationen“ (Euler und Hahn 2014, S. 78) reflektiert, bildet die bwp@ in ihrem Themenspektrum den Kern der Disziplin ab.

Hinzuweisen ist gesondert auf folgende Topics. Erstens ist Topic 2 („Projektberichte“) sehr stark im Datenkorpus repräsentiert. Dies mag ein Hinweis darauf sein, dass die Forschungspraxis in (zunehmenden) Maße projektförmig organisiert ist. Dies mag sich auf drittmittelgeförderte Forschungsprojekte oder Projekte mit der

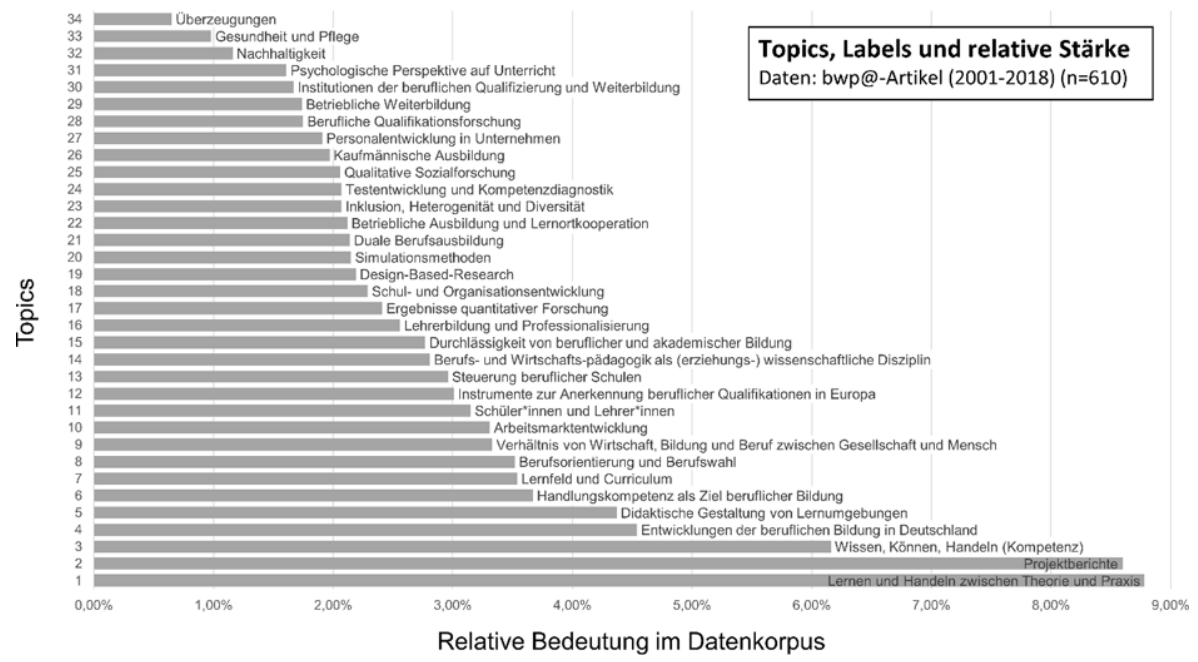

Abb. 6 Topics der computerlinguistischen Analyse 
schulischen/betrieblichen Praxis oder auf Projekte beziehen, zu denen sich mehrere Angehörige der Disziplin zeitweise zusammenschließen und korrespondiert schließlich auch mit dem Befund, dass Publikationen zunehmend kooperativ organisiert sind (s. oben). Zweitens sind einige Topics weniger inhaltlich, sondern eher forschungsmethodisch bzw. -paradigmatisch akzentuiert (z. B. Design-BasedResearch, Qualitative Sozialforschung, Testentwicklung und Kompetenzdiagnostik, Ergebnisse quantitativer Forschung). Damit kommt eine methodische Vielfalt zum Ausdruck, die die Berufs- und Wirtschaftspädagogik als eine empirisch arbeitende Disziplin kennzeichnet. Insofern bietet die bwp@ den Raum für unterschiedliche hermeneutische, qualitative und quantitative oder auch konstruktive Zugänge.

Abb. 6 veranschaulicht auch die relative Stärke der Topics im gesamten Datenkorpus. Auch einzelne Beiträge setzen sich immer aus mehreren Topics zusammen und streuen demnach verschiedene Themen in den Diskurs ein. Dies ist relevant für die Interpretation der Befunde, denn die in einem Beitrag identifizierten Topics sind somit nicht gleichbedeutend mit dem grundlegenden Thema des jeweiligen Artikels, sondern sind in ihm mehr oder weniger stark enthalten. Je fokussierter die Beträge, umso höher sind die Werte einzelner Topics $(\mathrm{Max}=73 \%)$ und die Varianz der Topics. Tab. 4 zeigt, dass es wenige Beiträge gibt, die eine dominante Fokussierung auf eine Thematik aufweisen (Gruppe I, 11\%). In dieser Gruppe prägt das stärkste der 34 möglichen Topics die Beiträge zu durchschnittlich 56\%. Auf der anderen Seite gibt es Beiträge, die sich durch eine kaum erkennbare Fokussierung bzw. positiv gewendet eine große thematische Breite auszeichnen (Gruppe IV, 6\%). Eine Bewertung dieses Befundes ist nicht ohne weiteres möglich, da die Topics bspw. auch methodisch akzentuiert sein können, sodass bei empirischen Arbeiten per se zwei Topics angesprochen sein können. ${ }^{5}$

Die Entscheidung, zunächst die bwp@ zu untersuchen, hatte neben dem einfachen Zugriff auf bereits digitalisierte Daten einen forschungsstrategischen Grund. Durch die Themengebundenheit der Ausgaben kann sie zur Validierung des Verfahrens und der identifizierten Topics genutzt werden. In einer Ausgabe zur Lehrkräfteausbildung (z.B. Ausgabe 2001/1) sollte das Topic „Lehrerbildung und Professionalität“ relativ stark sein, in einer zum curricularen Konzept des Lernfeldansatzes (z. B. Ausgabe

Tab. 4 Fokussierung der Beiträge

\begin{tabular}{|c|c|c|c|c|}
\hline Gruppe & Varianz & $\begin{array}{l}\text { MW stärkstes } \\
\text { Topic }(\%)\end{array}$ & $N$ & $\%$ \\
\hline $\begin{array}{l}\text { Gruppe I: Stark fokussierte } \\
\text { Beiträge }\end{array}$ & $\begin{array}{l}\text { Varianz über } \\
0,75 \%\end{array}$ & 56 & 69 & 11 \\
\hline $\begin{array}{l}\text { Gruppe II: mittel fokussierte } \\
\text { Beiträge }\end{array}$ & $\begin{array}{l}\text { Varianz zwischen } 0,50 \\
\text { und } 0,75 \%\end{array}$ & 40 & 159 & 26 \\
\hline $\begin{array}{l}\text { Gruppe III: weniger fokussierte } \\
\text { Beiträge }\end{array}$ & $\begin{array}{l}\text { Varianz zwischen } 0,25 \\
\text { und } 0,50 \%\end{array}$ & 28 & 344 & 56 \\
\hline $\begin{array}{l}\text { Gruppe IV: kaum fokussierte } \\
\text { Beiträge }\end{array}$ & $\begin{array}{l}\text { Varianz unter } \\
0,25 \%\end{array}$ & 18 & 38 & 6 \\
\hline Gesamt & Mittl. Varianz $=0,49 \%$ & 34 & 610 & 100 \\
\hline
\end{tabular}

\footnotetext{
5 Interessant wäre es in weiterführenden Analysen zu prüfen, welche Themen mit welchen forschungsmethodischen bzw. -paradigmatischen Zugriffen bearbeitet werden.
} 


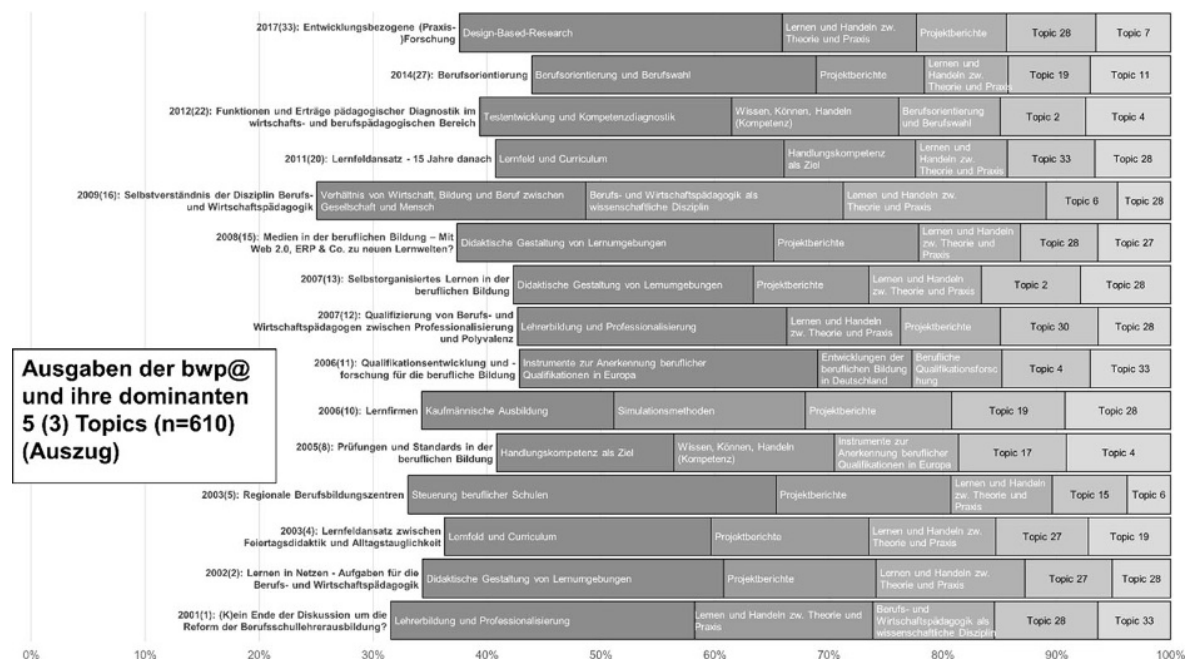

Abb. 7 Dominante Topics einzelner Ausgaben (Auszug)

2002/4) das Topic „Lernfeld und Curriculum“. Abb. 7 zeigt (in Auszügen), dass diese Zusammenhänge erwartungskonform bestehen und man von einem validen Verfahren ausgehen kann. Die einzelnen Hefte werden überwiegend gut durch die ersten 5 Topics beschrieben ( $\mathrm{Min}=40,4 \% ; \mathrm{Max}=74,9 \% ; \mathrm{M}=55,0 \% ; \mathrm{SD}=8,3 \%$ ).

\subsection{Identifikation sozial-kognitiver Strukturen}

Abschließend werden die Befunde zur sozialen und kognitiven Gestalt zusammengeführt. Es ist einerseits möglich, die dominanten Themen über verschiedene Artikel von Autoren*innen zu aggregieren. Damit kann man - hier eingeschränkt auf die Publikationen in der bwp@ - analysieren, wer über welche Themen kommuniziert. Analysiert man so die gesamte disziplinäre Kommunikation, kann man Protagonist*innen bestimmter Themen empirisch identifizieren. Allein mit den Daten aus dieser einen Zeitschrift ist dies weder möglich noch sinnvoll. Andererseits kann man die thematische Aggregation auch für Institutionen vornehmen und die Frage adressieren, welche Standorte (bzw. die ihnen zugehörigen Wissenschaftler*innen) über welche Themen (in der bwp@) kommunizieren und sich darüber hinaus in ihrer thematischen Ausrichtung ähneln.

Am gewählten Beispiel soll verdeutlicht werden, welche Forschungspotenziale diese Analyse beinhaltet. Es wird so ggf. möglich, disziplinäre, thematische Subgruppen und mithin ggf. verschiedene „Schulen“ (Kauder 2010) zu identifizieren, wenngleich hierzu neben den thematischen Aspekten weitere soziale und kognitive Aspekte zu integrieren sind.

Um thematische Ähnlichkeiten von Standorten und thematisch differenzierte Gruppen zu bestimmen, wurde mit einem Teil-Sample (nur Hochschulen und Forschungsinstitute mit min. 5 Autoren*innen) eine hierarchische Clusteranalyse (euklidische Distanz, ward-Algorithmus) durchgeführt. Zuvor wurde für jeden Standort 


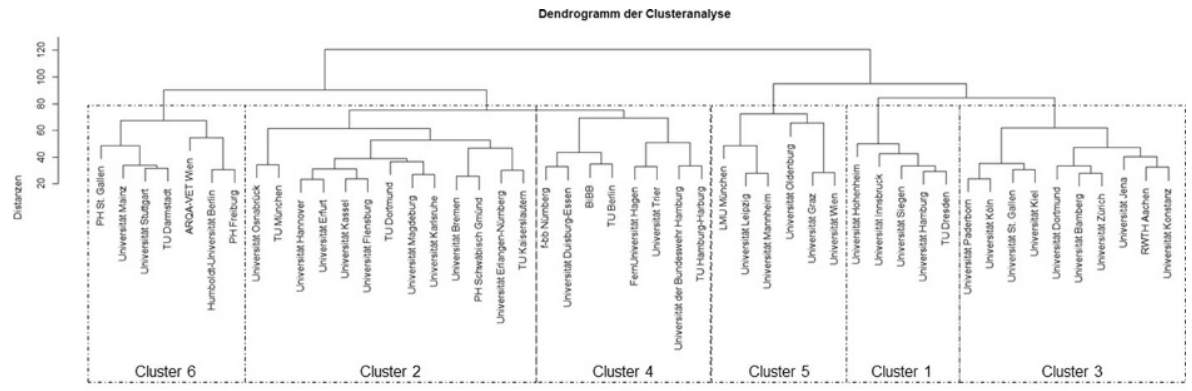

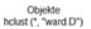

Abb. 8 Thematischen Ähnlichkeiten zwischen den Standorten (Ergebnis der hierarchischen Clusteranalyse)

über alle Artikel der ihm zugeordneten Autoren*innen der Mittelwert der Themen ermittelt. Als multivariates Analyseverfahren fasst die Clusteranalyse die Standorte dann derart zusammen, dass Gruppen gebildet werden können, deren Mitglieder sich einerseits ähnlich sind (Kohäsion) und die sich andererseits voneinander abheben (Separation) (Backhaus et al. 2000). Das Ergebnis dieser Analyse zeigt Abb. 8.

Vor diesem Hintergrund lassen sich bspw. 6 Gruppen von Standorten identifizieren, die inhaltlich an ähnlichen Themen arbeiten und sich zugleich mehr oder weniger stark von anderen Standorten unterscheiden. Diese sind mit ihren jeweiligen 5 dominanten Themen in Abb. 9 dargestellt. Die Autoren*innen der Standorte, die Cluster 6 zugeordnet werden (z. B. Stuttgart, Darmstadt, Mainz, Berlin) befassen sich stark mit „Testentwicklung und Kompetenzdiagnostik“, ,Schul- und Organisationsentwicklung“ sowie mit ,quantitativer Forschung“; Standorte aus Cluster 4 (z. B. Bundesinstitut für Berufliche Bildung (BiBB)) hingegen mit der „Entwicklung der beruflichen Bildung in Deutschland“, „Arbeitsmarktentwicklung“ oder „Fragen von

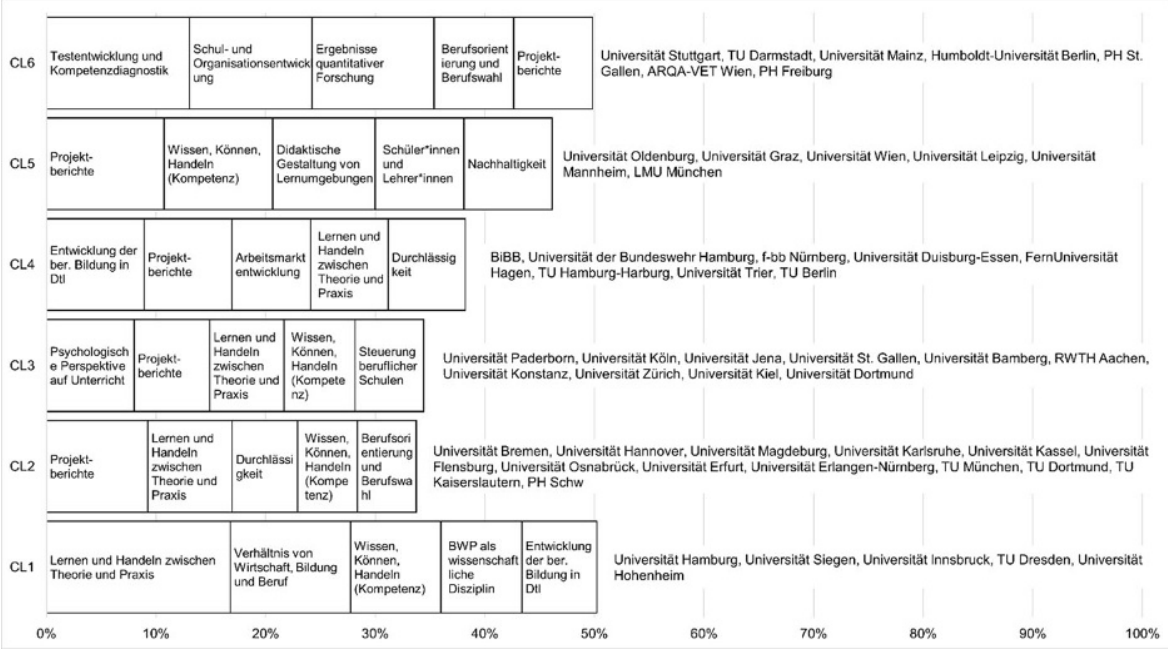

Abb. 9 Dominante Themen der Standortcluster 
Durchlässigkeit". Die beiden größeren Cluster 2 und 3 unterscheiden sich neben verschiedenen Topics auch dahingehend, dass CL 2 tendenziell starke berufspädagogische, CL 3 tendenziell eher wirtschaftspädagogische Standorte enthält. Man erkennt zudem, dass die inhaltliche Fokussierung der Gruppen mit ihrer Größe abnimmt und bestimmte Topics kaum Varianz erzeugen und von allen bearbeitet werden (z. B. Projektberichte, Lernen und Handeln zw. Theorie und Praxis).

Die Ergebnisse der Clusteranalyse sind plausibel, aber nicht vollständig. Auf dieser Datenbasis lässt sich noch kein abschließendes Bild der inhaltlichen Arbeitsfelder der Angehörigen dieser Standorte nachzeichnen, da offen ist, ob diese an anderen Stellen nicht über andere Themen kommunizieren - insb. weil die Ausgaben der bwp@ inhaltlich-thematische Fokussierung bereits vorgeben. Trotzdem ist dieses Verfahren geeignet, institutionelle (oder auch personelle) Ähnlichkeiten zu identifizieren und Daten zu generieren, die innerhalb einer Disziplin verschiedene „Schulen“ oder Paradigmengruppen abbilden. Offen ist, wie sich personelle Veränderungen (Emeritierungen und Neuberufungen) auf die kognitive Gestalt der Standorte auswirken.

\section{Zusammenfassung und Ausblick}

Die ganzheitliche Untersuchung wissenschaftlicher Disziplinen und ihrer Entwicklung erfordert verschiedene methodische Zugriffe auf diverse Gegenstände und Artefakte (Weingart und Winterhager 1984). In diesem Beitrag standen die Produzent*innen wissenschaftlicher Erkenntnisse im Fokus, deren Bedeutung seit Kuhn (1962) in der Wissenschaftsforschung unumstritten ist (Helm 1994, S. 170). Allerdings werden sie nicht als Individuen, sondern als Kollektiv - Community - analysiert und dessen Kommunikation (Götzl et al. 2018). Forschungsergebnisse von speziellen Fachleuten werden insb. in Fachzeitschriften publiziert (Papenkort 2015). Deren Entstehung und Existenz ist von hoher Bedeutung für die Entwicklung wissenschaftlicher Disziplinen (Clark 1972), sodass diese bestrebt sind, selbstverwaltete Kommunikationssysteme zu etablieren (Schriewer und Keiner 1993). Daher erlaubt ihre Analyse sowohl eine Beschreibung des Status Quo als auch disziplinärer Entwicklungen. Wer in disziplinären Fachzeitschriften kommuniziert, ist (wenn auch nur vorübergehend) Teil der Kommunikationsgemeinschaft und wie und worüber kommuniziert wird, Ausdruck der (sich ggf. wandelnden) disziplinären paradigmatischen Orientierungen.

Eine wissenschaftstheoretisch legitimierte Analyse disziplinärer Fachzeitschriften ist der Berufs- und Wirtschaftspädagogik nicht fremd (Kap. 2). Allerdings haben die herkömmlichen inhaltsanalytischen Verfahren, die insb. auf die ZBW angewandt wurden, methodische Schwächen. Daher wurde ein computerlinguistisches Verfahren des Topic Modeling eingesetzt und erprobt. Als Analysegegenstand fungierte erstmals exemplarisch die Zeitschrift „Berufs- und Wirtschaftspädagogik - online“, da sie aus genannten Gründen in besonderem Maße geeignet ist, das computerlinguistische Verfahren zu erproben und auf seine Validität hin zu prüfen (Kap. 3). Für die gesamte Disziplin repräsentative Befunde kann dieser Fokus auf ein Journal allerdings nicht liefern, was aber auch nicht der Anspruch des Beitrags ist. 
Die Analyse der sozialen Dimension hat ergeben, dass diese Fachzeitschrift durch eine Offenheit für viele Autor*innen der wissenschaftlichen Berufs- und Wirtschaftspädagogik geprägt ist - wobei festzustellen ist, dass keineswegs die gesamte Disziplin hier publiziert. Insofern ergibt die Analyse dieser Zeitschrift kein valides Gesamtbild der disziplinären Kommunikation, sondern fokussiert einen relevanten Ausschnitt dieser (4.1). Die computerlinguistische Analyse der kognitiven Dimension hat ein reichhaltiges Themenspektrum innerhalb der Zeitschrift offenbart, das relevante Gegenstände aber auch zentrale forschungsmethodische Zugriffe der Disziplin abbildet. Vor dem Hintergrund der zuvor genannten Limitationen bleibt jedoch offen, ob dieses thematische Spektrum die gesamte Disziplin hinreichend gut abbildet. Dies ist aber weniger eine Frage des Verfahrens, sondern des Gegenstands. Die inhaltliche Analyse zeigt zudem, dass innerhalb einzelner Ausgaben und vor allem innerhalb einzelner Beiträge eine thematische Varianz besteht. Dies reliabel quantifiziert abzubilden, ist eine große Stärke des vorgestellten Verfahrens. Gerade empirisch orientierte Abhandlungen verbinden gegenstandsbezogene Topics mit methodischen, die hier als getrennte Topics identifiziert werden. Die computergestützt identifizierten Themen weisen eine plausible Passung zu den Themen der einzelnen Ausgaben auf, was für die Validität des Ansatzes spricht. Eben dies prüfen zu können, war schließlich auch ein Grund für die Auswahl dieser Zeitschrift (4.2). Die Verbindung sozialer und inhaltlicher Aspekte erfolgte schließlich mittels einer Clusteranalyse. Diese hat thematische Ähnlichkeiten zwischen den Autor*innen verschiedener Standorte offenbart, sodass sich mit diesem Ansatz auch Gruppen innerhalb der disziplinären Gemeinschaft identifizieren lassen, die an ähnlichen Themen arbeiten bzw. über diese in einer Fachzeitschrift kommunizieren (4.3). Gerade dies ist aus wissenschaftstheoretischer Perspektive bedeutsam (Götzl et al. 2018): ,Sowohl die ,normale“ Wissenschaft als auch Revolutionen sind [...] gemeinschaftsbezogene Tätigkeiten. Um sie aufzuspüren und zu analysieren, muß man zuerst die sich wandelnde Gemeinschaftsstruktur der Wissenschaften entwirren. Ein Paradigma regiert zunächst nicht einen Gegenstandsbereich, sondern eine Gruppe von Fachleuten. Jede Untersuchung paradigma-gelenkter oder paradigma-zerstörender Forschung muß mit der Lokalisierung der verantwortlichen Gruppe oder Gruppen beginnen“ (Kuhn 1969, S. 191).

Einschränkend soll darauf hingewiesen sein, dass dieser computerlinguistische Zugriff und die hier vorgestellten Ergebnisse nicht völlig unabhängig von Entscheidungen der Forschenden sind. Dies beginnt bei der Auswahl des Gegenstands und der inkludierten Rubriken der Zeitschrift in der Stichprobe. Weiter ist das ,Labeling' der Topic-Listen eine (inter-)subjektive Interpretationsleistung und auch die Frage, wie viele Themen der Algorithmus ermittelt bzw. welche der verschiedenen Themenkonfigurationen oder -listen final gewählt wird, basiert auf Entscheidungen der Forschenden. Die Frage, wie mit welcher Konnotation über die Themen gesprochen wird, kann (zumindest an dieser Stelle) auch (noch) nicht beantwortet werden, wenngleich dies für die Ergebnisse hier auch nicht relevant ist - gleichwohl aber für weiterführende Untersuchungen.

Die durchgeführte Studie zeigt damit erstens, dass der hier vorgestellte methodische Ansatz erhebliche Potenziale für die thematisch-inhaltliche Analyse der disziplinären Kommunikation beinhaltet. Das besondere Potenzial für die (disziplinäre) 
Wissenschaftsforschung steckt vor allem in der empirischen Verbindung von inhaltlicher (Ideengestalt) und sozialer Dimension (Sozialgestalt). Auf dieser Basis lassen sich Gruppen mit unterschiedlichen paradigmatischen Orientierungen, Denkkollektive oder Schulen aufspüren. In längsschnittlicher Betrachtung können dann disziplinäre paradigmatische Entwicklungen, Prozesse von Wandel, Angleichung und Differenzierung sowie die damit verbundenen Interessen und Strukturen, Interaktions- und Aushandlungsprozesse oder gar Machtkämpfe untersucht werden sowie die Beziehungen zu und Einflüsse von weiteren gesellschaftlichen Subsystemen oder institutionellen Feldern (insb. Bildungs- und Wissenschaftspolitik oder andere Disziplinen). In weiterführenden Arbeiten ist mit dieser Art der Analyse auch das Verhältnis von wissenschaftlichen Institutionen und Personen zu untersuchen und u. a. zu analysieren, ob sich Institutionen bzw. Hochschulstandorte bei einem Wechsel der Wissenschaftler*innen verändern et vice versa.

Im Anschluss sollen nunmehr weitere disziplinäre Zeitschriften in einer längeren Zeitperspektive in die Analyse eingeschlossen werden, um ausgehend von diesem Beispiel einen umfassenderen Einblick in die disziplinäre Kommunikation zu gewinnen und paradigmatische Entwicklungen der Disziplin und einzelner Gruppen nachzeichnen zu können.

Funding Open Access funding enabled and organized by Projekt DEAL.

Open Access Dieser Artikel wird unter der Creative Commons Namensnennung 4.0 International Lizenz veröffentlicht, welche die Nutzung, Vervielfältigung, Bearbeitung, Verbreitung und Wiedergabe in jeglichem Medium und Format erlaubt, sofern Sie den/die ursprünglichen Autor(en) und die Quelle ordnungsgemäß nennen, einen Link zur Creative Commons Lizenz beifügen und angeben, ob Änderungen vorgenommen wurden.

Die in diesem Artikel enthaltenen Bilder und sonstiges Drittmaterial unterliegen ebenfalls der genannten Creative Commons Lizenz, sofern sich aus der Abbildungslegende nichts anderes ergibt. Sofern das betreffende Material nicht unter der genannten Creative Commons Lizenz steht und die betreffende Handlung nicht nach gesetzlichen Vorschriften erlaubt ist, ist für die oben aufgeführten Weiterverwendungen des Materials die Einwilligung des jeweiligen Rechteinhabers einzuholen.

Weitere Details zur Lizenz entnehmen Sie bitte der Lizenzinformation auf http://creativecommons.org/ licenses/by/4.0/deed.de.

\section{Literatur}

Ambrose, D., van Tassel-Baska, J., Coleman, L. J., \& Cross, T.L. (2010). Unified, insular, firmly policed, or fractured, porous, contested, gifted education? Journal for the Education of the Gifted, 33(4), 453-478.

Averbeck, S. (2008). Zur Methodologie fach- und theoriehistorischer Forschung. Triadischer Epistemologiebegriff. In K. Arnold, M. Brehmer \& B. Semrad (Hrsg.), Kommunikationsgeschichte. Positionen und Werkzeuge. Ein diskursives Hand-und Lehrbuch (S. 259-286). Berlin: Lit.

Backhaus, K., Erichson, B., Plinke, W., \& Weiber, R. (2000). Multivariate Analysemethoden. Berlin: Springer.

Baumann, J. M. (2009). Zur inhaltlichen Ausrichtung der universitären berufs- und wirtschaftspädagogischen Forschung in Österreich - zehn Jahre Forschungsdokumentation im Überblick. bwp@ Berufsund Wirtschaftspädagogik - online, 9(16), 1-22.

Blei, D. M. (2012). Probabilistic topic models. Communications of the ACM, 55(4), 77-84.

Bourdieu, P. (1984). Distinction: a social critique of the judgement of taste. Cambridge: Harvard University Press. 
Büchter, K., \& Gramlinger, F. (2001). Editorial bwp@1. https://www.bwpat.de/ausgabe1/editorialbwpatnr1.pdf. Accessed: 16 Aug 2021.

Büchter, K., Gramlinger, F., Kremer, H.-H., Tenberg, R., \& Tramm, T. (2011). bwp@ Berufs- und Wirtschaftspädagogik - online 2001-2011: Verortung, Selbstverständnis und Entwicklung. In: bwp@ Berufs- und Wirtschaftspädagogik - online, Jubiläumsausgabe, 1-11. http://www.bwpat.de/10jahre_ bwpat/buechter_etal_10jahre-bwpat.pdf. Accessed: 27. Nov 2011.

Clark, T.N. (1972). The stages of scientific institutionalization. International Social Science Journal, 24(4), 658-671.

Clark, T.N. (1974). Die Stadien wissenschaftlicher Institutionalisierung. In P. Weingart (Hrsg.), Wissenschaftssoziologie (S. 105-121). Frankfurt a. M.: Fischer.

Cretchley, J., Rooney, D., \& Gallois, C. (2010). Mapping a 40-year history with Leximancer: themes and concepts in the Journal of Cross-Cultural Psychology. Journal of Cross-Cultural Psychology, 41(3), 318-328.

DiMaggio, P. J., Nag, M., \& Blei, D. (2013). Exploiting affinities between topic modeling and the sociological perspective on culture: application to newspaper coverage of U.S. government arts funding. Poetics, 41(6), 570-606.

Euler, D., Hahn, A. (2014). Wirtschaftsdidaktik. 3., aktualisierte Aufl. Bern: Haupt.

Felt, U., Nowotny, H., \& Taschwer, K. (1995). Wissenschaftsforschung. Eine Einführung. Frankfurt a. M.: Campus.

Fleck, L. (1980). Entstehung und Entwicklung einer wissenschaftlichen Tatsache: Einführung in die Lehre vom Denkstil und Denkkollektiv. Frankfurt a. M.: Suhrkamp.

Goldenstein, J., \& Poschmann, P. (2019a). Analyzing meaning in big data: performing a map analysis using grammatical parsing and topic modeling. Sociological Methodology, 49(1), 83-131.

Goldenstein, J., \& Poschmann, P. (2019b). Rejoinder: a quest for transparent and reproducible text-mining methodologies in computational social science. Sociological Methodology, 49(1), 144-151.

Goldenstein, J., Poschmann, P., \& Walgenbach, P. (2019). Die Erfassung und Messung von Bedeutungsstrukturen in großen Textsammlungen: Die Nutzung neuartiger Werkzeuge des Natural Language Processing im Neo-Institutionalismus. In R. Hasse \& A. K. Krüger (Hrsg.), Neo-Institutionalismus Kritik und Weiterentwicklung eines sozialwissenschaftlichen Paradigmas (S. 215-246). Bielefeld: transcript.

Götzl, M., Geiser, P., \& Jahn, R. W. (2018). Zur Institutionalisierung der Berufs- und Wirtschaftspädagogik im 20. Jahrhundert. Ein kollektivbiographischer und netzwerkanalytischer Beitrag zur Etablierung der Wirtschaftspädagogik als (erziehungs-)wissenschaftliche (Teil-)Disziplin. In K. Vogel, C. Bers, J. Brauns, A. Hild, A. Stisser \& K.-P. Horn (Hrsg.), Windungen und Wendungen in der Erziehungswissenschaft (S. 107-122). Bad Heilbrunn: Julius Klinkhardt.

Hannigan, T.R., Haans, R.F. J., Vakili, K., Tchalian, H., \& Glaser, V.L. (2019). Topic modeling in management research: rendering new theory from textual data. Academy of Management Annals, 13(2), 586-632.

Harney, K., Bormann, K., \& Wehrmeister, F. (1994). Das Berufsbildungssystem als Erschwernis der Berufs-und Wirtschaftspädagogik: Strukturelle Dilemmata und Möglichkeiten der Zeitschrift für Berufs-und Wirtschaftspädagogik (ZBW). In K. Stratmann (Hrsg.), Berufs- und wirtschaftspädagogische Zeitschriften. Aufsätze zu ihrer Analyse (S. 383-395). Frankfurt a. M.: GAFB.

Helm, L. (1994). Wer gehört zum Korpus der Erziehungswissenschaft? Konzeptionelle Überlegungen und forschungspraktische Konsequenzen. In K.-P. Horn \& L. Wigger (Hrsg.), Systematiken und Klassifikationen in der Erziehungswissenschaft (S. 169-188). Weinheim: Beltz.

Hentke, R. (1982). Zur Wissenschaftssystematik der Berufs- und Wirtschaftspädagogik. Reaktivierung und Weiterentwicklung verdrängter Konzeptionen. Zeitschrift für Berufs- und Wirtschaftspädagogik, 78(1), 17-30.

Jahn, R.W., Borkowski, T. P., \& Götzl, M. (2019). Strukturelle und inhaltliche Entwicklung der ZBW in den Jahren 2000-2016. Zeitschrift für Berufs- und Wirtschaftspädagogik, 115(4), 644-673.

Kaesler, D. (1984). Die frühe deutsche Soziologie 1909 bis 1934 und ihre Entstehungsmilieus. Eine wissenschaftssoziologische Untersuchung. Opladen: Westdt. Verlag.

Kauder, P. (2010). Wissenschaftliche Schulen in der Erziehungswissenschaft. Exemplarische und explorative Annäherungen an ein kaum erforschtes Thema. Zeitschrift für Pädagogik, 56(4), 564-581.

Keiner, E. (1999). Erziehungswissenschaft 1947-1990: Eine empirische und vergleichende Untersuchung zur kommunikativen Praxis einer Disziplin. Weinheim: Dt. Studien-Verl..

Kipp, M., \& Miller-Kipp, G. (2004). ZBW-Geschichtsschreibung - (k)ein Feld für Legendenbildung? Zeitschrift für Berufs- und Wirtschaftspädagogik, 100(4), 529-534. 
Klusmeyer, J. (1999). Die Entwicklung der Berufs- und Wirtschaftspädagogik in ihrem Fachschriftentum. In A. Schelten, P.F.E. Sloane \& G. A. Straka (Hrsg.), Berufs- und Wirtschaftspädagogik im Spiegel der Forschung. Forschungsberichte des DGfE-Kongresses 1998 (S. 11-26). Wiesbaden: VS.

Klusmeyer, J. (2001). Zur kommunikativen Praxis der Berufs- und Wirtschaftspädagogik in ihrem Fachschrifttum Ein Beitrag zu formalen, sozialen und kognitiven Selbstreflexionsaspekten der Berufs- und Wirtschaftspädagogik anhand einer Inhaltsanalyse der ,Zeitschrift für Berufs- und Wirtschaftspädagogik" (Beiträge zur Berufs- und Wirtschaftspädagogik). Oldenburg: Universität Oldenburg.

Klusmeyer, J. (2002a). Zur Rekonstruktion von forschungsmethodischen Entwicklungstrends in der Berufs- und Wirtschaftspädagogik im Spiegel ihres Fachschrifttums. Zeitschrift für Berufs- und Wirtschaftspädagogik, 98(2), 161-180.

Klusmeyer, J. (2002b). Zur kommunikativen Praxis der Berufs- und Wirtschaftspädagogik in ihrem Fachschrifttum. In M. Eckert, M. Horlebein, I. Lisop, H. Reinisch \& T. Tramm (Hrsg.), Bilanzierungen. Schulentwicklung, Lehrerbildung und Wissenschaftsgeschichte im Feld der Wirtschafts- und Berufspädagogik (S. 151-163). Frankfurt a. M.: GAFB.

Klusmeyer, J. (2010). Forschungsmethodische Grundorientierungen in der Berufs- und Wirtschaftspädagogik und in ihrem Fachschrifttum. In R. Nickolaus, G. Pätzold, H. Reinisch \& T. Tramm (Hrsg.), Handbuch Berufs- und Wirtschaftspädagogik (S. 383-385). Bad Heilbrunn: Julius Klinkhardt.

Klusmeyer, J., Reinisch, H., \& Söll, M. (2011). Wo publizieren Berufs- und Wirtschaftspädagogen? Eine Vorstudie zur Erfassung des Publikationsverhaltens in der Berufs- und Wirtschaftspädagogik. Zeitschrift für Berufs- und Wirtschaftspädagogik, 107(3), 328-350.

Kuhn, T. (1976). Die Struktur wissenschaftlicher Revolutionen. 2., rev. u. um das Postskriptum von 1969 erg. Aufl. Frankfurt am Main: Suhrkamp.

Kobayashi, V.B., Mol, S. T., Berkers, H. A., Kismihók, G., \& Den Hartog, D. N. (2018). Text mining in organizational research. Organizational Research Methods, 21(3), 733-765.

Krohn, W., \& Küppers, G. (1989). Die Selbstorganisation der Wissenschaft. Frankfurt a. M.: Suhrkamp.

Kuhn, T.S. (1962). The structure of scientific revolutions. Chicago: University of Chicago Press.

Kuhn, T. S. (2014). Die Struktur wissenschaftlicher Revolutionen (24. Aufl.). Frankfurt a. M.: Suhrkamp.

Lepenies, W. (1981). Einleitung. Studien zur kognitiven, sozialen und historischen Identität der Soziologie. In W. Lepenies (Hrsg.), Geschichte der Soziologie. Studien zur kognitiven sozialen und historischen Identität einer Disziplin (S. 1-14). Frankfurt a. M.: Suhrkamp.

Liesch, P. W., Håkanson, L., McGaughey, S. L., Middleton, S., \& Cretchley, J. (2011). The evolution of the international business field: a scientometric investigation of articles published in its premier journal. Scientometrics, 88(1), 17-42.

Lin, J., \& Lee, S. T. (2012). Mapping 12 years of communication scholarship: themes and concepts in the Journal of Communication. In D. Hutchison \& T. Kanade (Hrsg.), The outreach of digital libraries: a globalized resource network (S. 359-360). Berlin: Springer.

Macke, G. (1989). Rekonstruktion von Wissenschaftsentwicklung. Analyse der methodischen Grundorientierung erziehungswissenschaftlicher Qualifikationsarbeiten. In W. Bos \& C. Tarnai (Hrsg.), Angewandte Inhaltsanalyse in empirischer Pädagogik und Psychologie (S. 86-101). Münster: Waxmann.

Martin, J. L. (2003). What is field theory? American Journal of Sociology, 109(1), 1-49.

Mohr, J. W., \& Bogdanov, P. (2013). Introduction topic models: what they are and why they matter. Poetics, 41(6), 545-569.

Muller, M., Guha, S., Baumer, E. P. S., Mimno, D., \& Shami, N. S. (2016). Machine learning and grounded theory method. In Association for Computing Machinery (Hrsg.), Proceedings of the 19th International Conference on Supporting Group Work (GROUP '16, S. 3-8). New York: ACM.

Nelson, L. K. (2017). Computational grounded theory: a methodological framework. Sociological Methods \& Research, 49(1), 3-42.

Nelson, L. K., Burk, D., Knudsen, M., \& McCall, L. (2018). The future of coding: a comparison of handcoding and three types of computer-assisted text analysis methods. Sociological Methods and Research. https://doi.org/10.1177/0049124118769114.

Papenkort, U. (2015). Darstellungen der Pädagogik. Bibliografie deutschsprachiger Lehr-, Hand-, und Wörterbücher 1945 bis 2012. In P. Kauder \& P. Vogel (Hrsg.), Lehrbücher der Erziehungswissenschaft - ein Spiegel der Disziplin (S. 15-31). Bad Heilbrunn: Julius Klinkhardt.

Pätzold, G. (2004). Einhundert Jahrgänge ZBW: zwischen Beständigkeit und Wandel einer berufs- und wirtschaftspädagogischen Zeitschrift. Zeitschrift für Berufs- und Wirtschaftspädagogik, 100(4), 481-487.

Reinisch, H. (2006). Berufspädagogische Zeitschriften. In F.-J. Kaiser \& G. Pätzold (Hrsg.), Wörterbuch Berufs- und Wirtschaftspädagogik (S. 159-161). Bad Heilbrunn: Klinkhardt. 
Reinisch, H. (2009). Über Nutzen und Schaden des Philosophierens über das Selbstverständnis der Berufsund Wirtschaftspädagogik. Anmerkungen aus wissenschaftssoziologisch inspirierter Sicht. bwp@ Berufs- und Wirtschaftspädagogik - online, 9(16), 1-17.

Reinisch, H. (2010). Geschichtsschreibung und Disziplinbildung: Anmerkungen zur Funktion der frühen Histographie des kaufmännischen Bildungswesens im Prozess der Etablierung der Wirtschaftspädagogik als Wissenschaft. In E. Wuttke \& K. Beck (Hrsg.), Was heißt und zu welchem Ende studieren wir die Geschichte der Berufserziehung? (S. 181-199). Opladen: Budrich.

Schriewer, J., \& Keiner, E. (1993). Kommunikationsnetze und Theoriegestalt: Zur Binnenkonstitution der Erziehungswissenschaft in Frankreich und Deutschland. In J. Schriewer (Hrsg.), Sozialer Raum und akademische Kulturen. Studien zur europäischen Hochschul- und Wissenschaftsgeschichte im 19. und 20. Jahrhundert (S. 277-341). Frankfurt a. M.: Peter Lang.

Söll, M., Reinisch, \& Klusmeyer, H. J. (2014). Publikation und Reputation. Eine Studie zum Rezeptionsund Publikationsverhalten von wissenschaftlich tätigen Berufs- und Wirtschaftspädagoginnen und -pädagogen. Zeitschrift für Berufs- und Wirtschaftspädagogik, 110(4), 505-528.

Stichweh, R. (1984). Zur Entstehung des modernen Systems wissenschaftlicher Disziplinen - Physik in Deutschland 1740 - 1890. Frankfurt a. M.: Suhrkamp.

Stratmann, K. (1992). Hundert Jahre ZBW oder von den wechselnden Funktionen einer berufs- und wirtschaftspädagogischen Zeitschrift. Zeitschrift für Berufs- und Wirtschaftspädagogik, 88(8), 619-622.

Taubert, N. C., \& Weingart, P. (2010). Open Access: Wandel des wissenschaftlichen Publikationssystems. In T. Sutter \& A. Mehler (Hrsg.), Medienwandel als Wandel von Interaktionsformen (S. 159-181). Wiesbaden: VS.

Tenberg, R., Eder, A., \& Sterrenberg, K. (2009). Struktur und Qualität wissenschaftlicher Aufsätze in der ZBW. Inhaltsanalyse der ZBW-Jahrgänge 2000 bis 2008. Zeitschrift für Berufs- und Wirtschaftspädagogik, 105(3), 418-427.

Weingart, P., \& Winterhager, M. (1984). Die Vermessung der Forschung: Theorie und Praxis der Wissenschaftsindikatoren. Frankfurt a. M.: Campus.

Zabeck, J. (1978). Paradigmenpluralismus als wissenschaftliches Programm - Ein Beitrag zur Überwindung der Krise der Erziehungswissenschaft. In W. Brand \& D. Brinkmann (Hrsg.), Tradition und Neuorientierung in der Berufs- und Wirtschaftspädagogik (S. 291-332). Hamburg: Wiechelmann.

Zabeck, J. (1998). Die Berufs- und Wirtschaftspädagogik als erziehungswissenschaftliche Teildisziplin. In D. Hoffmann \& K. Neumann (Hrsg.), Die gegenwärtige Struktur der Erziehungswissenschaft. Zum Selbstverständnis einer undisziplinierten Disziplin (S. 171-185). Weinheim: Dt. Studien-Verlag.

Zawacki-Richter, O., \& Naidu, S. (2016). Mapping research trends from 35 years of publications in Distance Education. Distance Education, 37(3), 245-269.

Zietsma, C., Groenewegen, P., Logue, D. M., \& Hinings, C. R. (2017). Field or fields? Building the scaffolding for cumulation of research on institutional fields. Academy of Management Annals, 11(1), $391-450$. 